\title{
A Predictive Energy Management Strategy for Multi- Mode Plug-in Hybrid Electric Vehicles Based on Multi Neural Networks
}

\author{
Yitao $\mathrm{Wu}^{1}$, Yuanjian Zhang ${ }^{2}$, Guang $\mathrm{Li}^{3}$, Jiangwei Shen ${ }^{1}$, Zheng Chen ${ }^{1,3^{*}}$ and Yonggang $\mathrm{Liu}^{4 *}$ \\ ${ }^{1}$ Faculty of Transportation Engineering, Kunming University of Science and Technology, Kunming, 650500, China \\ ${ }^{2}$ Sir William Wright Technology Center, Queen's University Belfast, Belfast, BT9 5BS, United Kingdom \\ ${ }^{3}$ School of Engineering and Materials Science, Queen Mary University of London, London, E1 4NS, United \\ Kingdom \\ ${ }^{4}$ State Key Laboratory of Mechanical Transmissions \& School of Automotive Engineering, Chongqing University, \\ Chongqing, 400044, China \\ Email: wuyitaomail@qq.com,y.zhang@qub.ac.uk,g.li@qmul.ac.uk,shenjiangwei6@163.com, chen@kust.edu.cn, \\ andylyg@umich.edu \\ Corresponding Authors: Zheng Chen (chen@kust.edu.cn) and Yonggang Liu (andylyg@umich.edu)
}

Abstract: Online optimal energy management of plug-in hybrid electric vehicles has been continually investigated for better fuel economy. This paper proposed a predictive energy management strategy based on multi neural networks for a multi-mode plug-in hybrid electric vehicle. To attain it, firstly, the offline optimal results prepared for knowledge learning are derived by dynamic programming and Pontryagin's minimum principle. Then, the mode recognition neural network is trained based on the optimal results of dynamic programming and the recurrent neural network is firstly exploited to realize online co-state estimation application. Consequently, the velocity prediction-based online model predictive control framework is established with the co-state correction and slacked constraints to solve the real-time optimal control sequence. A series of numerical simulation results validate that the optimal performance yielded from global optimal strategy can be exploited online to attain the satisfied cost reduction, compared with equivalent consumption minimum strategy, with the assistance of estimated real time co-state and slacked reference. In addition, the computation duration of proposed algorithm decreases by $23.40 \%$, compared with conventional Pontryagin's minimum principle-based model predictive control scheme, thereby proving its online application potential.

Key Words: Plug-in hybrid electric vehicles, model predictive control, dynamic programming, neural network, Pontryagin’s minimum principle.

\section{INTRODUCTION}

In correspondence with the requirements of progressively rigorous environment protection and energy savings, development of high-efficient environmental-friendly vehicles has emphasized its significance in transport industry [1]. Vehicle electrification takes an important role in lessening dependence on fossil energy 
and mitigating air pollution [2]. Pure electric vehicles (PEVs), however, are still under a long way of perfection and marketization, thanks to the well-known shortcomings of long charging duration and range anxieties arisen by limited charging capacity and energy density of batteries [3, 4]. Hybrid electric vehicles (HEVs) and plugin HEVs (PHEVs) are both treated as potential alternatives to extend the driving range by merging co-operation of electric motor and internal combustion engine (ICE). The main difference between HEV and PHEV lies in that the latter is equipped with a larger battery pack, thus supplying a certain all-electric range (AER) [5], whereby PHEV can be regarded a mixture of conventional HEV and PEV. Thus, the advantages of PEV and conventional HEV are integrated together to compensate both limitations, while incurring further difficulties of allocating the energy distribution between two energy sources, compared with HEV and PEV, which is often tackled by energy management strategies (EMSs) [6, 7].

To attain better energy allocation for economic driving, a variety of EMSs have been developed by industry and academia, and they can be mainly classified into three categories: rule-based methods, optimization-based methods and artificial intelligence-based methods. As a truism, rule-based methods (such as charge depletion/charge sustaining (CD/CS) scheme) are widely employed in PHEVs due to their simplicity and ease of application, direct interpretation and high reliability [8,9]. Nonetheless, their optimal solutions cannot be easily attained; and furthermore, a well-calibrated set of rules usually entail considerable efforts during formulation of implicit rules and threshold parameters [10]. Based on the wavelet transform, Ref. [11] divides propelling power into the high frequency and low frequency segments, and respectively allocates them to super capacitors and battery pack, thereby mitigating the battery's degradation rate arisen by large and frequent current variation and simultaneously attaining higher operating efficiency. In [12], a threshold-based rule table is constructed to attain torque distribution for a PHEV, and a correction model is introduced to pursue optimality according to the predicted traffic information. Ref. [13] proposes a combined EMS for HEVs, which includes three stages - electric driving, charge depletion and charge sustaining; and dynamic programming (DP) is employed to find optimal results to calibrate the engine's working region for improving online operating performance [14]. Although rule-based strategies enable easy real-time implementation, considerable efforts and engineering practice are indispensable for further improvement of overall performance.

Optimization based strategies, which can be divided into two types - offline and online, generally cost considerable computational effort to search/find the solution, and lead to optimal/near-optimal control 
performance regarding the targeted cost function [15]. Offline optimization methods often necessitate comprehensive driving knowledge when finding optimal solutions [16]. Obviously, the methods are inappropriate for practical application, whereas the yielded solutions can be usually considered as benchmarks for evaluating performances of other algorithms. DP is well-suited for solving optimization problems with existence of multi-stage decisions, and is effective in dealing with nonlinear time-varying optimization problems [17-19]. In [17], DP is leveraged to acquire the optimal solution under identified different driving conditions, followed by the extraction of online strategy. Pontryagin's minimum principle (PMP) is also a representative alternative for solving optimal control problems [20, 21]. Ref. [22] applies the PMP to find the global optimal solution and demonstrates that the optimal control can be attained by a constant equivalent factor, given the assumption that the battery open circuit voltage and internal resistance are independent of state of charge (SOC). In [23], by considering the aging process of battery, the optimal depth of discharge (DOD) is investigated in detail and the battery size and EMS are synergistically optimized by convex programming (CP) for a PHEV. Although global optimality of mentioned approaches can be reached, detailed information with respect to whole driving conditions are usually required in advance. Online algorithms such as model predictive control (MPC) $[24,25]$ and equivalent consumption minimum strategy (ECMS) [26] are popular for overcoming shortcomings of offline optimization methods. MPC shows flexibility in coping with real-time constraints as well as online optimization and is progressively applied in energy management of PHEVs. In [27], a hierarchical predictive EMS is proposed, in which the sequential quadratic programming (SQP) is employed in the MPC based framework to gain the optimal trade-off between fuel cell life and energy cost. In [28], the optimal DOD is planned by PMP considering battery's degradation rate, and then the MPC is employed to find the optimal energy distribution scheme at each time step. Ref. [29] proposes a map-based ECMS to alleviate the calculation intensity, in which the equivalent factor is determined by particle swarm optimization (PSO) under different driving conditions and different initial SOC. In particular, PMP-based MPC shows strong adaptation to different driving condition and highlights better computational efficiency. However, a challenging task is that in each time step, the initial co-state cannot be determined directly, thus resulting in redundant iterative calculation of online optimization.

Artificial intelligence-based methods have recently been considered as competitive candidates for online energy management without requirements of deterministic models [30, 31]. In [32], two neural networks (NNs) 
are trained to recognize the road type and driving habit, respectively; and another two NNs are trained on the basis of DP results to provide real-time battery power and engine speed commands. This approach benefits from the strong ability that $\mathrm{NN}$ can learn implicit knowledge from optimal solutions that is undetectable and difficult to be extracted manually. Ref. [33] proposes a real-time EMS based on a high-order Markov predictor and radial basis function NN (RBFNN), in which fifteen variables characterizing driving patterns as well as the offline optimal engine working points are imported to train the model, such that RBFNN can suggest the working region of the engine online. The EMS proposed in [34] builds up a two-hidden-layer NN to generate nearoptimal power commands, in which the space domain trip information - the percentage of driving length over the total range is introduced for the NN training to reduce uncertainty of real-time transportation. Along with other input characters, the proposed NN with two hidden layers are built up to output near-optimal power commands. Reinforcement learning (RL) has been intensively investigated and applied to address the adaptive optimization in EMS according to driving pattern and driving habits [14, 35]. Ref. [36] proposes a RL-based EMS for PHEV, and the Markov process is introduced to find the transition probability under a series of different standard driving cycles, and the Q-learning algorithm is employed to find the optimal controlling sequence. The results indicate that a maximum $10.1 \%$ reduction of total cost can be attained, compared with the CD/CS method. Generally speaking, this type of approaches shows model-free and heuristic advantages during the development process, thereby reducing human efforts to some extent and improving the self-adaptiveness; whereas, how to translate the learned information into online control needs to be further investigated.

As discussed above, although some actual rules can be extracted from global optimum to guide real-time power allocations of PHEVs, complexity and uncertainty still exist in real driving scenarios; on the other hand, it is difficult to acquire the comprehensive knowledge from global optimal solution. In this context, the battery SOC in real applications is usually difficult to be controlled in the same, or even similar, manner as the optimal solution does, and feedback control is often furnished to enable a superior track of the SOC trajectory. In terms of online approaches for SOC feedback, such as MPC and PMP-based real-time strategies, the planned SOC trajectory can be tracked; nonetheless the co-state or equivalent factor, that is difficult to be determined, often incurs redundant calculation without predicted knowledge of the whole trip. A possible approach is to combine some useful global knowledge extracted from DP or PMP results when designing the predictive EMS. To incorporate all the discussed issues and further improve the calculation efficiency, an online NN reinforced 
predictive EMS, as shown in Fig. 1, is herein developed for a specially designed PHEV, of which the powertrain features multiple operation modes controlled by two clutches and one brake. As can be seen from Fig. 1, the optimal solutions are attained offline by global optimization algorithms including DP and PMP under different driving cycles. DP, that is more effective in coping with discretized mode control, accounts for the discrete optimization of energy management in a certain trip with easy application; while PMP is leveraged to explore more valuable information (mainly the co-state) to provide a reliable and convenient manner for online calculation of battery power. If the variation law in view of the co-state can be extracted by the PMP, then the co-state can be relatively easy to be found in unfamiliar driving conditions. Thus, DP and PMP are leveraged in this study for collection of global optimal solutions. Consequently, given a proper co-state value at each time step, the battery power can be determined only by minimizing the Hamilton function. By this manner, the costate estimation-based method of calculating battery power can be more direct and easier to be implemented, compared with DP. In the premise of attaining the destination at the beginning of a predetermined driving task, three NNs are respectively trained with respect to the operating modes of the vehicle powertrain, the co-state and the vehicle's speed prediction, thus relieving the online optimization labor and paving the road for application of the MPC. Finally, all the references, together with the vehicle's current status will be inputted into the MPC algorithm, thereby achieving the online locally optimal energy management in a rolling time horizon with the help of planed SOC trajectory and slacked constraint. The main contributions of this research can be summarized into the following three aspects: (1) a real-time energy management strategy for multi-mode PHEVs based on multi NNs and MPC algorithm is elaborated; (2) the recurrent neural network (RNN) is, to the authors' knowledge, firstly introduced to predict the co-state for online PMP calculation, and the accuracy and prediction performance is justified; and (3) the global and local SOC profiles based on the trip distance are merged not only for provision of online reference, but also for slacked constraints, thereby avoiding unnecessary and excessive tracking. To sum up, the proposed EMS framework can make full use of global information to ensure the optimality in real time, and additionally enable stable and fast online implementation, thus highlighting its online application potential.

The remainder of the paper is arranged as follows: In Section II, a multi-mode target PHEV is introduced and modeled in detail. Section III implements the global optimization based on DP and PMP; and meanwhile, the NNs in terms of mode, co-state and speed prediction are investigated. The overall working framework along 
with the MPC calculation is described in Section IV, followed by the validation and analysis discussed in Section V. Finally, the main conclusions and future research work are drawn in Section VI.

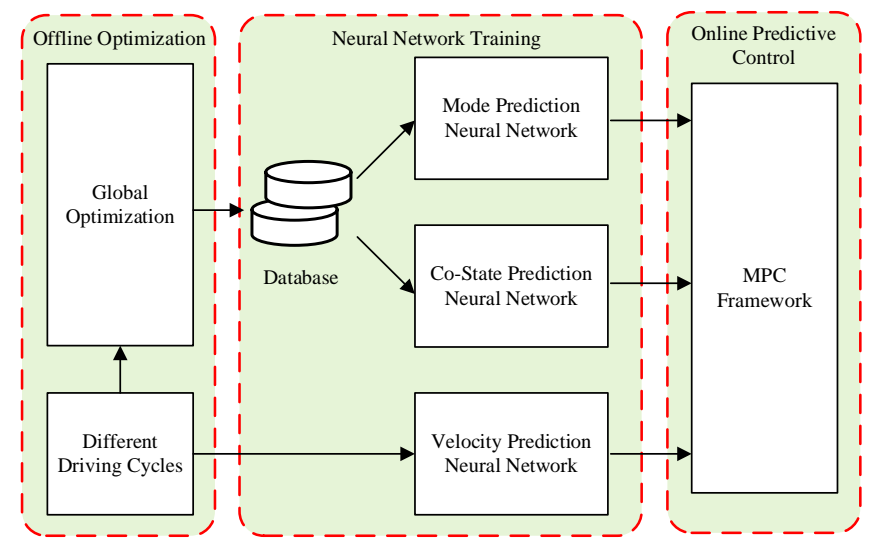

Fig. 1. The proposed EMS framework.

\section{THE POWERTRAIN MODELING}

In this study, a PHEV with a multi-mode powertrain, as illustrated in Fig. 2, is investigated for energy management [37]. The powertrain is composed of a planetary gear device, an engine, a battery pack, two traction motors (denoted as motor 1 and motor 2), two clutches (namely clutch 1 and clutch 2), one brake and other electric accessories. Motor 1 is always connected to the sun gear, and motor 2 is both connected to clutch 1 and clutch 2 . The carrier of the planetary gear set is responsible for supplying the driving power. Proper release and engagement of clutches and brake can result in different operating modes. To route legal power transmission, we need to notice that: 1) Release of clutch 1 can cut off the power delivered from the engine so that the pure electric drive can be attained. 2) The brake can terminate the rotation between the ring gear and the chassis; however, the clutch 2 attempts to deliver energy from/to the ring gear, thus clutch 2 and brake always operate oppositely. According to the status of clutches 1 and 2, two electric drive modes, namely single-motor mode and dual-motor mode, and two hybrid drive modes, i.e., the series mode and power-split mode can be defined and referred to as mode I to IV hereinafter. The corresponding clutch and brake states with regard to the four operating modes are listed in Table I, in which " 0 " represents the released state and " 1 ” expresses the engaged state for the clutch or brake. The crank shaft output is connected to clutch 1 , by which the engine power can be directly delivered to motor 2 and the ring gear depending on the state of clutch 2. 


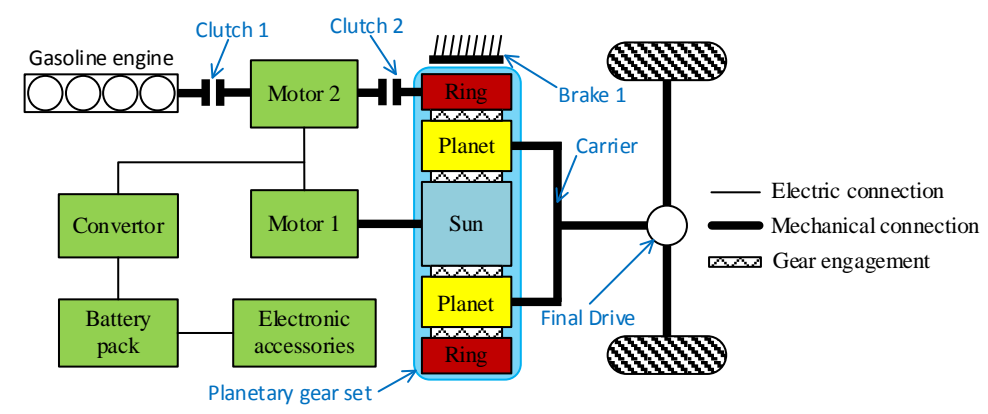

Fig. 2. The vehicle powertrain structure.

Table I. The clutch and brake states on each mode.

\begin{tabular}{llll}
\hline Mode & Clutch 1 state & Clutch 2 state & Brake \\
\hline One-motor EV (I) & 0 & 0 & 1 \\
Two-motors EV (II) & 0 & 1 & 0 \\
Series (III) & 1 & 0 & 1 \\
Power-split (IV) & 1 & 1 & 0 \\
\hline
\end{tabular}

From Fig. 2 and Table I, the speed and torque relationship from the planetary gear set to wheel will differ according to different power delivery routes. As to the modes I and III, the motor 1 is the only power source, and the mechanical transmission relationship can be expressed as:

$$
\begin{cases}T_{r}=\frac{\rho T_{c}}{1+\rho} & \omega_{r}=0 \\ T_{s}=\frac{T_{c}}{1+\rho} & \omega_{s}=\omega_{c}(1+\rho)\end{cases}
$$

where $\rho$ denotes the gear ratio of sun gear to ring gear of the planetary set, $T$ and $\omega$ express the torque and speed of one component, and the subscripts $r, c$ and $s$ denote the ring, carrier and sun gear of the planetary set, respectively. As to the modes II and IV, the torque and speed delivered via clutch 2 are coupled to the output, thus

$$
\left\{\begin{array}{l}
T_{r}=\frac{\rho T_{c}}{1+\rho} \\
T_{s}=\frac{T_{c}}{1+\rho}
\end{array} \omega_{c}=\frac{\omega_{s}}{1+\rho}+\frac{\rho \omega_{r}}{1+\rho}\right.
$$

A gasoline ICE with the maximum power of $73 \mathrm{~kW}$ is deployed in the vehicle. Note that the dynamic characteristics is often neglected for ease of EMS design, and hence the hot fuel map of ICE is constructed for simplicity to quantify the fuel rate based on the quasi-static data. In addition, to make full use of ICE and reduce the controlling degree of freedom (DOF), an optimal operating line (OOL), as shown in Fig. 3, is introduced to guide the engine's operation [24]. That is to say, the ICE works only in a preset deterministic mode with paired speed and torque when the output power is fixed. By this manner, the DOF can be decreased from two to one. 
A lithium-ion battery pack with 66 cells connected in series serves as the electric energy storage system. To simplify the design of EMS, an equivalent first-order circuit model is employed to characterize the dynamic and static electrical performance of battery. On this basis, the battery current $I$ can be calculated, as:

$$
I=\frac{V_{o c}-\sqrt{V_{o c}^{2}-4 R_{i n} P_{b a t}}}{2 R_{i n}}
$$

where $P_{b a t}$ denotes the battery power, $V_{o c}$ denotes the open circuit voltage; and $R_{i n}$ represents the internal resistance. It should be noticed that $V_{o c}$ and $R_{i n}$ generally vary with battery SOC during the driving process. The battery SOC, represented by $x$, is chosen as the state variable in this paper and defined as:

$$
x=\frac{Q-\int I d t}{Q}
$$

where $Q$ represents the nominal capacity of the battery pack in Ampere-hour (Ah). By combing (3) and (4), the state function can be yielded, as:

$$
\dot{x}=-\frac{I}{Q}=-\frac{V_{o c}-\sqrt{V_{o c}^{2}-4 R_{i n} P_{b a t}}}{2 Q R_{i n}}
$$

In addition, the other main parameters of the target vehicle are summarized in Table II.

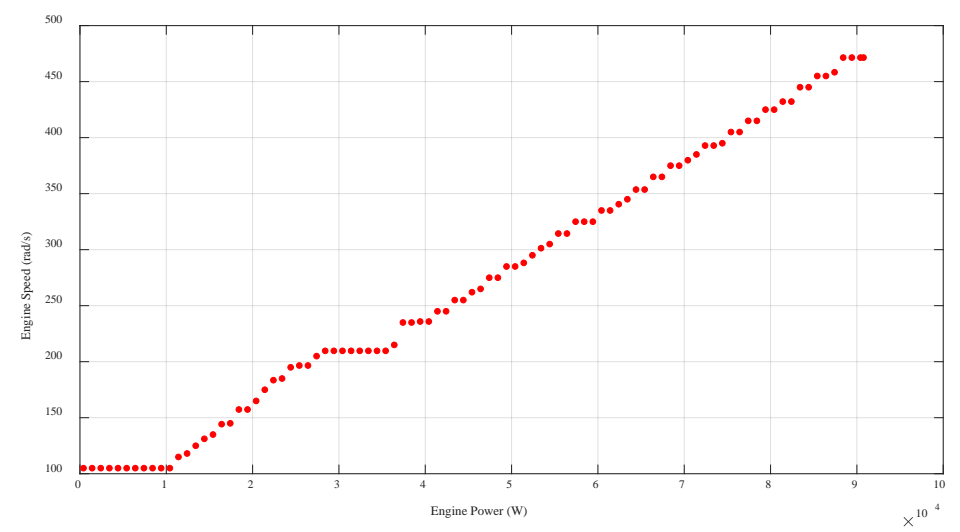

Fig. 3. The engine OOL.

Table II. Vehicle parameters.

\begin{tabular}{lll}
\hline Components & Parameters & Value \\
\hline Motor 1/2 & Peak Power & $70 \mathrm{~kW} / 50 \mathrm{~kW}$ \\
Planetary Gear Set & Ring Gear Teeth & 83 \\
Final Drive & Sun Gear Teeth & 37 \\
Wheel & Gear Ratio & 3.02 \\
\multirow{2}{*}{ Lithium-ion Battery Pack } & Radius & $0.3 \mathrm{~m}$ \\
Total Mass & Rated Capacity & $47.03 \mathrm{Ah}$ \\
& Rated Voltage & $233.89 \mathrm{~V}$ \\
\hline
\end{tabular}




\section{ELABORATION OF EMS DESIGN}

In this section, firstly, the optimization target of energy management is defined; and then the design of EMS is elaborated, which is summarized as five modules, i.e., the DP and PMP application for offline results, the NN-based driving mode recognition, the RNN-based co-state estimation, the future speed prediction based on RBFNN as well as the online MPC application with slacked constraints.

\section{A. Problem Definition and Algorithm Introduction}

In this study, the total cost in a certain trip including the fuel and charging, as expressed in (6), is considered as the optimization target. Thereinto, $\dot{m}_{f}\left(P_{b a t}(t), M(t)\right)$ denotes the instantaneous fuel consumption, which is related to $P_{b a t}$ and operating mode $M$, and $t_{0}$ and $t_{f}$ represent the initial and ending time of trip, respectively. $\varphi\left(x\left(t_{f}\right)\right)$ reflects the charging cost generated when charging the battery to the initial SOC at the end of a trip. To guarantee fair evaluation of fuel and electricity consumption, the fuel cost is transformed into the actual cost (\$) by multiplying the gasoline price $\gamma_{f}$, as:

$$
J=\gamma_{f} \int_{t_{0}}^{t_{f}} \dot{m}_{f}\left(P_{b a t}(t), M(t)\right) d t+\varphi\left(x\left(t_{f}\right)\right)
$$

When $P_{b a t}$ and $M$ are selected as the control inputs, the optimal problem can be formulated, as:

$$
\begin{aligned}
& \left\{\begin{array}{l}
\min J=\gamma_{f} \int_{t_{0}}^{t_{f}} \dot{m}_{f}\left(P_{b a t}(t), M(t)\right) d t+\varphi\left(x\left(t_{f}\right)\right) \\
\dot{x}=-\frac{V_{o c}-\sqrt{V_{o c}^{2}-4 R_{\text {in }} P_{\text {bat }}}}{2 Q R_{\text {in }}} \quad x(0)=x_{0}
\end{array}\right. \\
& \text { s.t. }\left\{\begin{array}{l}
P_{e n g}^{\min } \leq P_{e n g} \leq P_{e n g}^{\max } \\
P_{b a t}^{\min } \leq P_{b a t} \leq P_{b a t}^{\max } \\
M \in\{\mathrm{II}, \mathrm{III}, \mathrm{IV}\} \\
T_{m o t 1}^{\min } \leq T_{\text {mot1 }} \leq T_{m o t 1}^{\max } \\
T_{\text {mot } 2}^{\min } \leq T_{\text {mot2 }} \leq T_{\text {mot } 2}^{\max } \\
\omega_{m o t 1}^{\min } \leq \omega_{m o t 1} \leq \omega_{\text {mot1 }}^{\max } \\
\omega_{m o t 2}^{\min } \leq \omega_{\text {mot2 }} \leq \omega_{m o t 2}^{\max } \\
S O C^{\min } \leq X \leq S O C^{\max }
\end{array}\right.
\end{aligned}
$$

where $P_{\text {eng }}$ represents the engine power; $x_{0}$ denotes the initial SOC value; $T_{\text {mot } 1}$ and $T_{\text {mot } 2}$ express the torque of motors 1 and 2; and $\omega_{\text {mot1 }}$ and $\omega_{\text {mot2 }}$ denote their rotational speed, respectively. In addition, the superscripts min and max indicate the lower and upper limitations of corresponding variables. It should be noted 
that the modes I and II will not result in any instantaneous fuel consumption; in other word, they do not make any difference on the optimizing result but incur heavier computation. Therefore, the modes I and II are treated as the same scenario during optimization.

The detailed EMS architecture to solve the problem is summarized in Fig. 4, where the global optimizations including DP and PMP are leveraged to find the optimal data sets firstly. In general, DP is adopted to solve discretized multi-stage optimization problems, while PMP excels at solving a problem in continuous time horizon. In this research, the operating modes are treated as four discretized states. Owing to the discrete operating modes and the discrete engine on/off commands, DP is the most suitable solver to guarantee the calculation precision and optimization. In contrast, offline PMP is sensitive to continuity and derivativity of the Hamilton equation and state function, which conflicts with the target optimization problem with the discretized characteristics. To this end, DP is firstly adopted to solve the original problem in a discretized way, and then given the obtained optimal mode and optimal battery discharge depth solved by DP, PMP can be engaged in a simpler manner to explore the optimal co-state trajectory. Sequentially, three NNs are leveraged to supply the optimal parameters and predictive information for real-time control; and finally, the MPC is applied to cope with the local optimization. In addition, twelve standard driving cycles, including CYCLE505, HWFET, IM240, JC08, LA92, NEDC, REP05, SC03, UDDS, US06, WLTC and WVUSB, and two customized cycles, namely KM1 and KM2 tested in Kunming, China, plotted in Fig. 5, are involved to assist in designing and validating the proposed strategy. It is worth noting that in the proposed EMS, the driver's destination is presumed known to the controller at the beginning of a trip with the assistance of global position system (GPS) and geographic information system (GIS), and the historical travel information can be accumulated, thus the remaining distance are available during driving to provide simple traffic information. Moreover, the NNs involved in this paper are constructed and trained in Matlab/Simulink. 


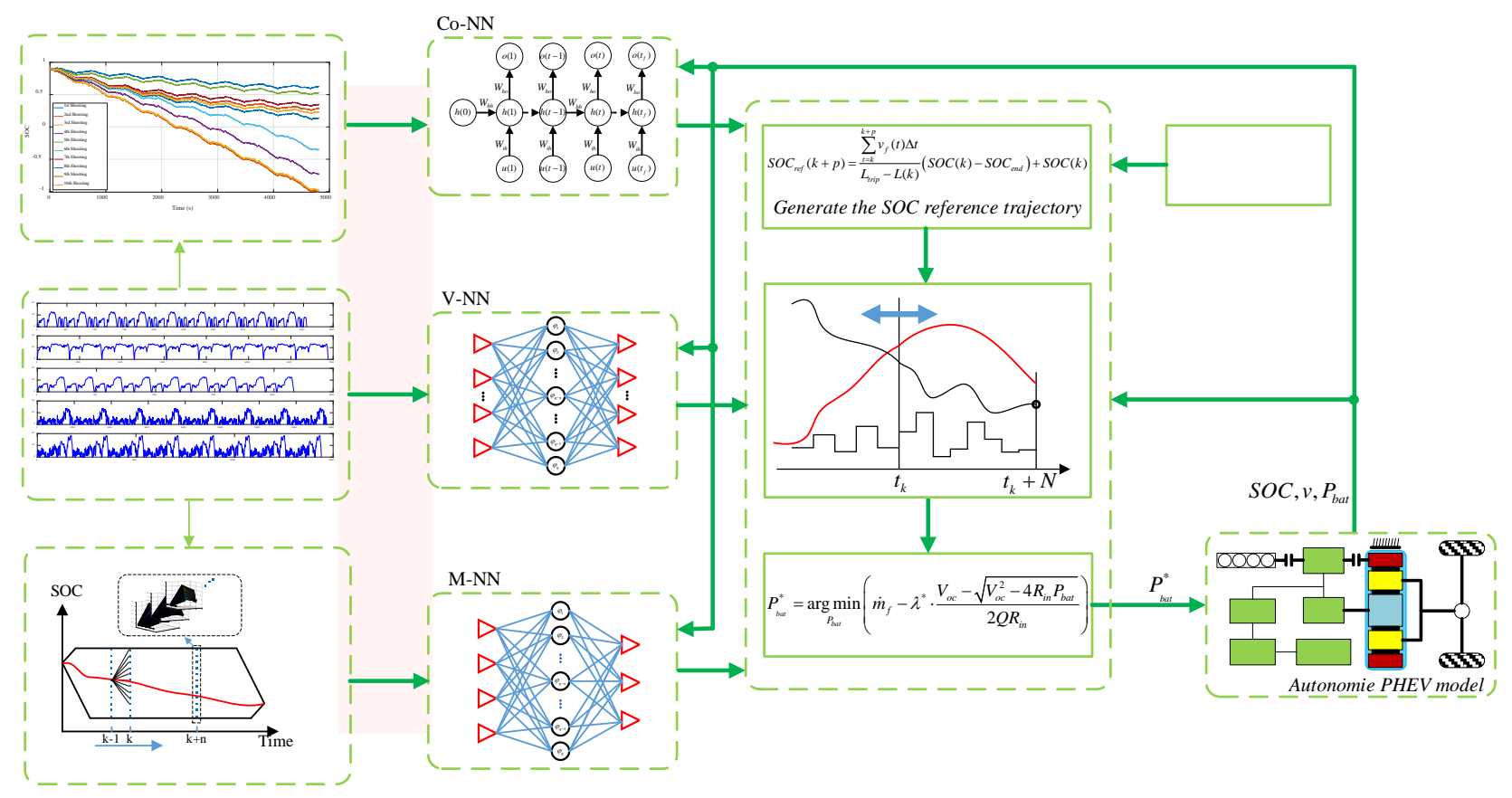

Fig. 4. The EMS development architecture.
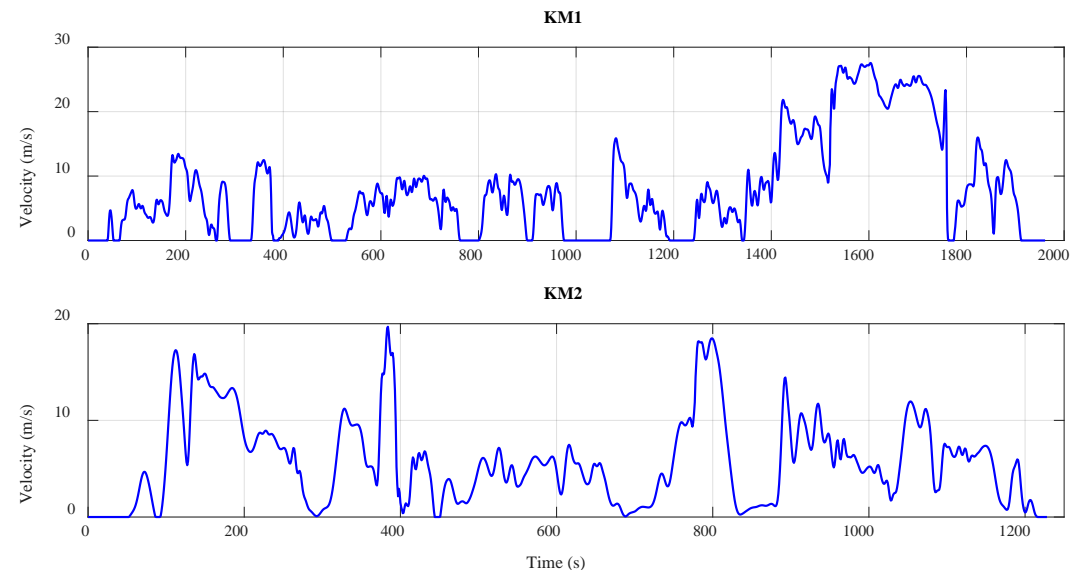

Fig. 5. Real word driving cycles.

\section{B. Application of Dynamic Programming}

In this study, DP that is well-suited for multi-state decision optimization is leveraged to find the offline global optimal solution. The kernel description of DP can be formulated as:

$$
\left(P_{b a t}^{*}(j), M^{*}(j)\right)=\underset{\left[P_{b a t}(j), M(j)\right] \in \Omega(j)}{\arg \min } \dot{m}_{f}\left(P_{b a t}(j), M(j)\right)+J^{*}(j+1)
$$

where $\Omega(j)$ denotes the admissible control set at time step $j$, the superscript * denotes that the corresponding variables are the optimal candidates. From (8), DP needs to solve the problem in time domain from the ending moment, as the solution at step $j$ depends on the solution of step $j+1$. A general knowledge is that DP is able to find the optimal solution but is not feasible in dealing with real-time control problem. However, comprehensive global optimal solutions among referred driving cycles can be treated as training data set and evaluation 
benchmark. Fig. 6 (a) shows the optimal results of SOC and operating mode solved by DP under five repetitive WLTC cycles, and Fig. 6 (b) shows the corresponding controlling sequences of battery power. The initial SOC $x_{0}$ is set to $90 \%$ and ends at $23.3 \%$. The result indicates that by controlling the battery power, the SOC trajectory declines averagely, by and large, as the trip goes on, and the engine is started when the mode IV is activated due to demand of more propelling power. To further find the intrinsic variation laws of SOC variation, Fig. 7 shows all of the 88 SOC trajectories in terms of optimizations based on different cycle types and lengths. It needs to be indicated that the optimization problem is defined with a terminal state cost rather than a certain terminal state; however, the results identically highlight that the battery SOC terminates in a relatively fixed region, of which the average value is $24.9 \%$. That is to say, given the predefined free terminal state optimization problem with final state cost, there exists an optimal terminal state, i.e., the optimal DOD, when attaining optimal energy management of target vehicle. In other words, the energy management with unconstrained terminal states can be converted to that with a fixed terminal state. Thereby, this simplification eliminates the final cost term in (6), by which the total cost can merely equal the total fuel consumption.

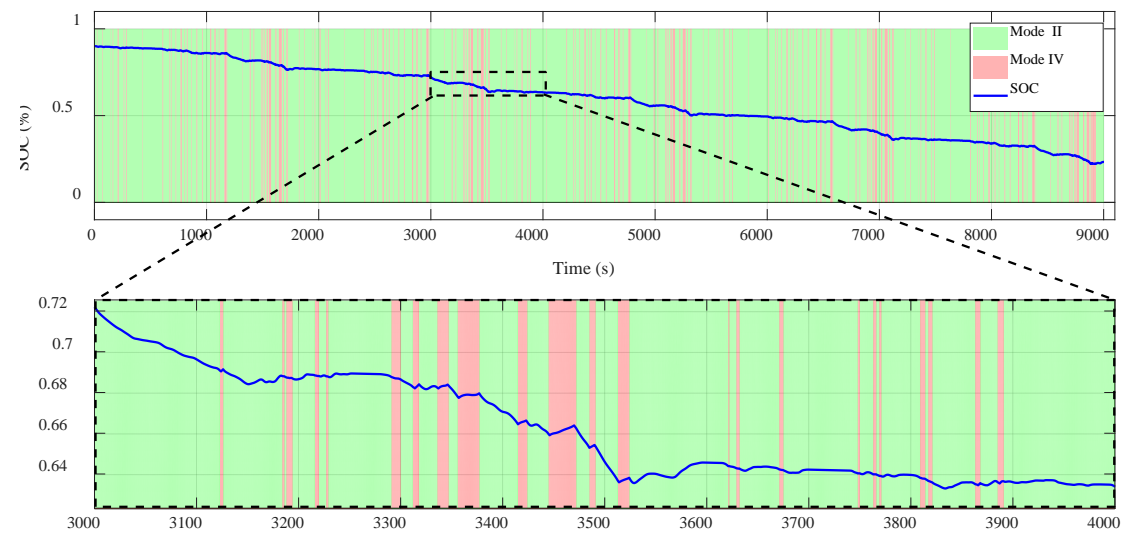

(a)

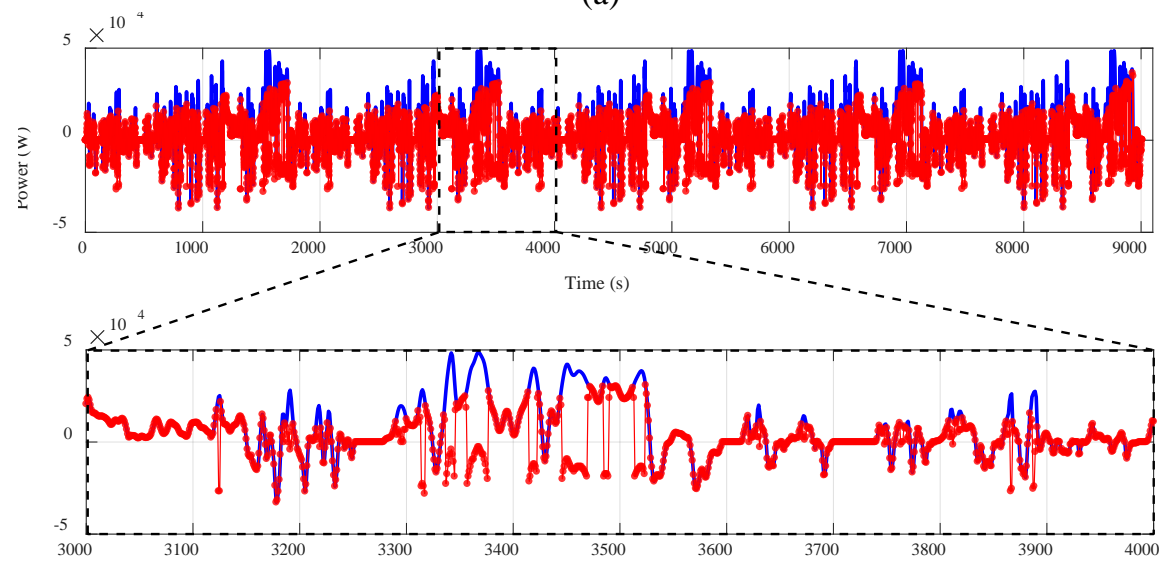

Fig. 6. The DP results.

(b) 


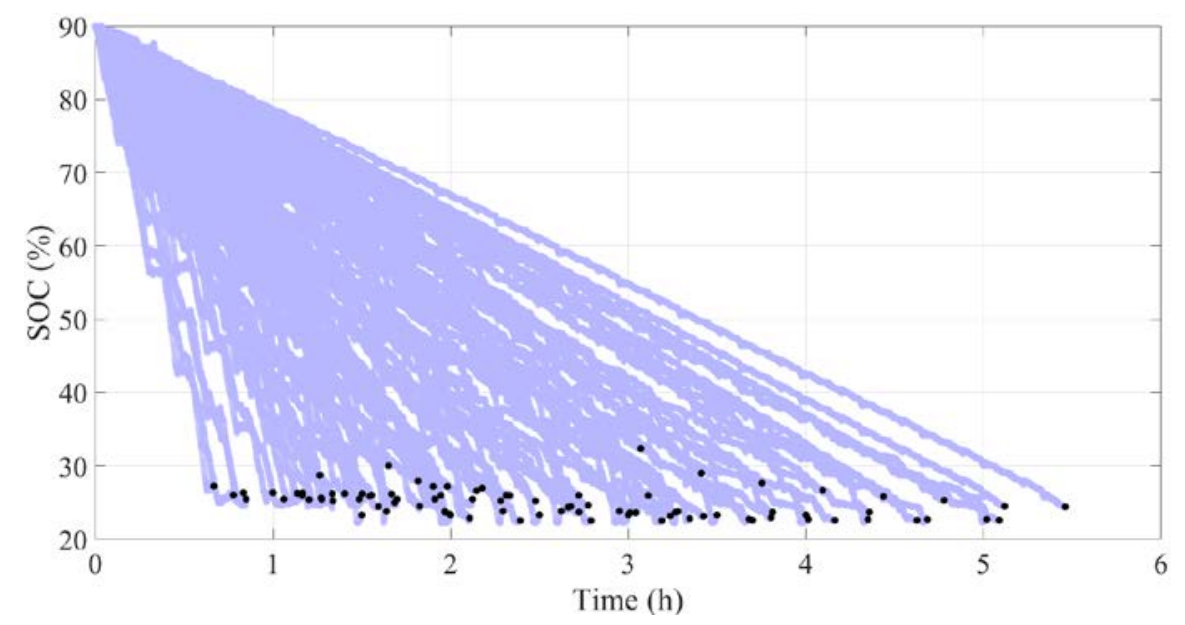

Fig. 7. The terminal SOC of DP. The black dot at the end of each trajectory marks its ending point.

With regard to operating mode, Fig. 8 shows the operating modes with respect to vehicle speed, demanded power and battery SOC. The modes II, III and IV respectively occupy $90.13 \%, 0.07 \%$ and $9.8 \%$, implying that the mode II dominates in all the trips, the mode IV is triggered when more driving power that is beyond the battery's current capability should be furnished by the engine in terms of optimality, and the mode III is seldom activated. On the other hand, according to the distribution map, the mode II attempts to work at lower speed, lower demanded power and regenerative braking, while the mode IV endeavors to work at higher speed and higher demanded power scenario. The mode III dispersedly appears in the edge of other modes. Owing to the sparse distribution of mode III, it can be neglected when further simplifying the design of strategy.

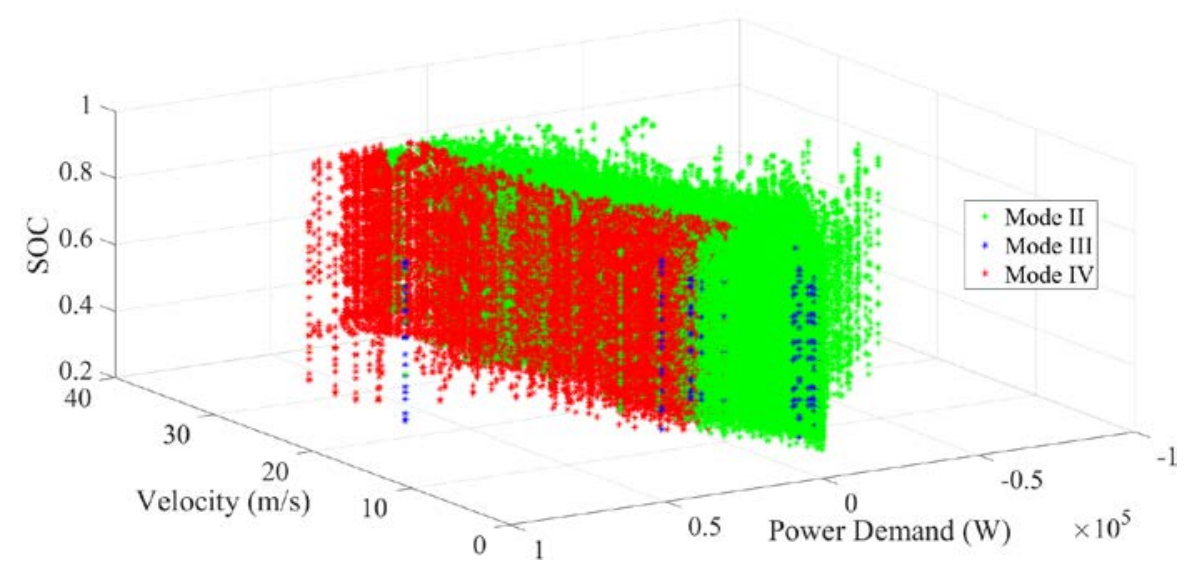

Fig. 8. Operating mode distribution.

\section{Driving Mode Recognition Based on Neural Network}

The task of proposed research is to develop an online strategy according to the offline optimal solution, and it is imperative to establish a mode predictor according to the real-time driving information. In this study, a 
three-layer feedforward NN with a sigmoid hidden layer and a softmax output layer, referred to as M-NN, is served as the online mode predictor. The input features of mode recognition NN include the battery SOC, vehicle speed, demanded power and engine ratio, of which the latter is defined as:

$$
E r=\frac{L_{\text {remain }}}{S O C_{\text {remain }}}=\frac{L\left(t_{f}\right)-L(t)}{x(t)-x\left(t_{f}\right)}
$$

where $L_{\text {remain }}$ denotes the remaining trip length, $S O C_{\text {remain }}$ means the remaining SOC, and $L(t)$ represents the traveled trip length at time $t$. With the development of GPS and GIS, $L\left(t_{f}\right)$ can be easily acquired, and thus it can be regarded as the prior knowledge. From (9), it can be recognized that $E r$ implies the degree of battery participation over the remaining distance. If the trip length is large while the battery capacity remains low, $E r$ is large, indicating more frequent engine operation, and meanwhile less battery power will be supplied, and vice versa. On this account, it can contribute to classification of the mode distribution for different trips. In addition, the optimal results by DP under CYCLE505, HWFET, IM240, JC08, LA92, NEDC and REP05 with different repetitions are employed as the training data set, and the remaining cycles are for validation. A feedforward NN with 1 hidden layer and 17 hidden neurons is finally established for this work.

\section{Application of Pontryagin’s Minimum Principle}

Similar with the mode prediction, the battery power can be calculated if the optimal knowledge can be extracted from DP results. Unfortunately, as shown in Fig. 6 (b), the battery power changes frequently and even stochastically, leading to difficulties in reformulating a simple relationship with respect to the battery power. From the intuitional view, the fitting and classification-based method is not preferred to solve the problem. Instead, PMP can attain extra sets of optimal control information for the battery power. As discussed previously, the optimal DOD is taken as the terminal state, thus the terminal cost term in (6) can be removed in the PMP's solving process. By introducing a co-state, the Hamilton function can be formulated, as:

$$
H=\gamma_{f} \dot{m}_{f}\left(P_{b a t}(t), M(t)\right)-\mu(t) \frac{V_{o c}-\sqrt{V_{o c}^{2}-4 R_{i n} P_{b a t}}}{2 Q R_{\text {in }}}
$$

where $\mu$ represents the co-state. The state equation can be formulated as:

$$
\left\{\begin{array}{l}
\dot{x}=\frac{\partial H}{\partial \mu}=-\frac{V_{o c}-\sqrt{V_{o c}^{2}-4 R_{i n} P_{b a t}}}{2 Q R_{i n}} \\
\dot{\mu}=-\frac{\partial H}{\partial x}=-\mu \frac{\partial \dot{x}}{\partial x}
\end{array}\right.
$$


Furthermore, the co-state dynamic in (11) can be updated to:

$$
\begin{aligned}
\dot{\mu} & =-\frac{\mu}{2 Q}\left(\frac{V_{o c} \frac{d V_{o c}}{d x}-2 P_{b a t} \frac{d R_{i n}}{d x}}{R_{i n} \sqrt{V_{o c}^{2}-4 R_{\text {in }} P_{b a t}}}-\frac{\left.\sqrt{V_{o c}^{2}-4 R_{\text {in }} P_{b a t}} \frac{d R_{\text {in }}}{d x}-\frac{1}{R_{i n}} \frac{d V_{o c}}{d x}+\frac{V_{o c}}{R_{i n}{ }^{2}} \frac{d R_{\text {in }}}{d x}\right)}{}=-\frac{\mu}{2 Q}\left(\frac{V_{o c}+\sqrt{V_{o c}^{2}-4 R_{\text {in }} P_{b a t}}}{R_{\text {in }} \sqrt{V_{o c}^{2}-4 R_{\text {in }} P_{b a t}}} \frac{d V_{o c}}{d x}+\frac{V_{o c} \sqrt{V_{o c}^{2}-4 R_{\text {in }} P_{b a t}}+2 R_{\text {in }} P_{b a t}-V_{o c}^{2}}{R_{\text {in }} \sqrt{V_{o c}^{2}-4 R_{\text {in }} P_{b a t}}} \frac{d R_{\text {in }}}{d x}\right)\right.
\end{aligned}
$$

From (12), the co-state changes with $d V_{o c} / d x$ and $d R_{\text {in }} / d x$, which can be obtained by the polynomial fitting and interpolation, as depicted in Fig. 9.
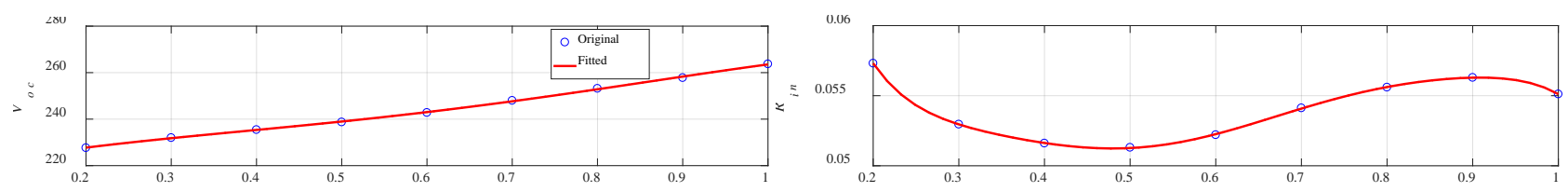

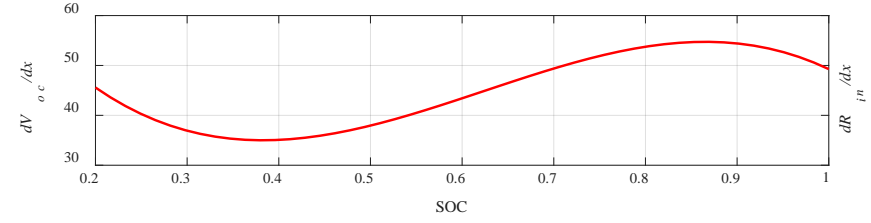

(a)

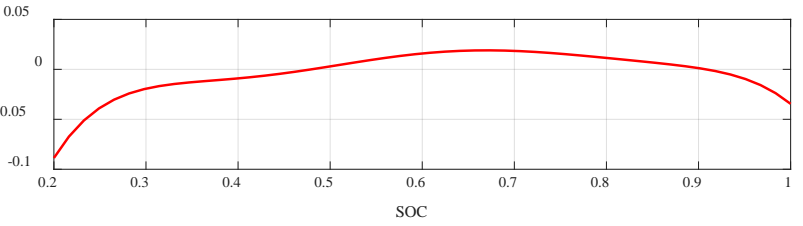

(b)

Fig. 9. The fitting results of open circuit voltage and internal resistance. (a) Voltage and its derivative; (b) Resistance and its derivative.

The constraints imposed on PMP are the same as those of DP, and the boundary conditions are reformulated as:

$$
\left\{\begin{array}{l}
x(0)=x_{0}=0.9 \\
x\left(t_{f}\right)=x_{t f}=0.249
\end{array}\right.
$$

where $x_{t f}$ represents the final state constraint according to the optimal DOD. Given an optimal $\mu(t)$, the battery power for minimum cost should satisfy:

$$
H\left(P_{b a t}(t), x^{*}(t), \mu^{*}(t)\right) \geq H\left(P_{b a t}^{*}(t), x^{*}(t), \mu^{*}(t)\right)
$$

The necessary condition in (14) can be explained as follows. On the optimal path of both SOC and co-state, the optimal control enables the Hamilton function to reach the minimum value at $t$. To this end, the well-known shooting method is adopted to solve the two-point boundary problem, of which the calculation process is illustrated in Fig. 10, and the corresponding results are shown in Fig. 11. By assuming that the co-state can be calculated in a determined trip, the optimal battery power can be indirectly resolved by (14). Different from battery power, the co-state changes monotonically with time, and thus can be treated as time-series signals 
during the trip, which is favorable for recognition. Here, the RNN is introduced to learn the intrinsic law concealing in the co-state data set.

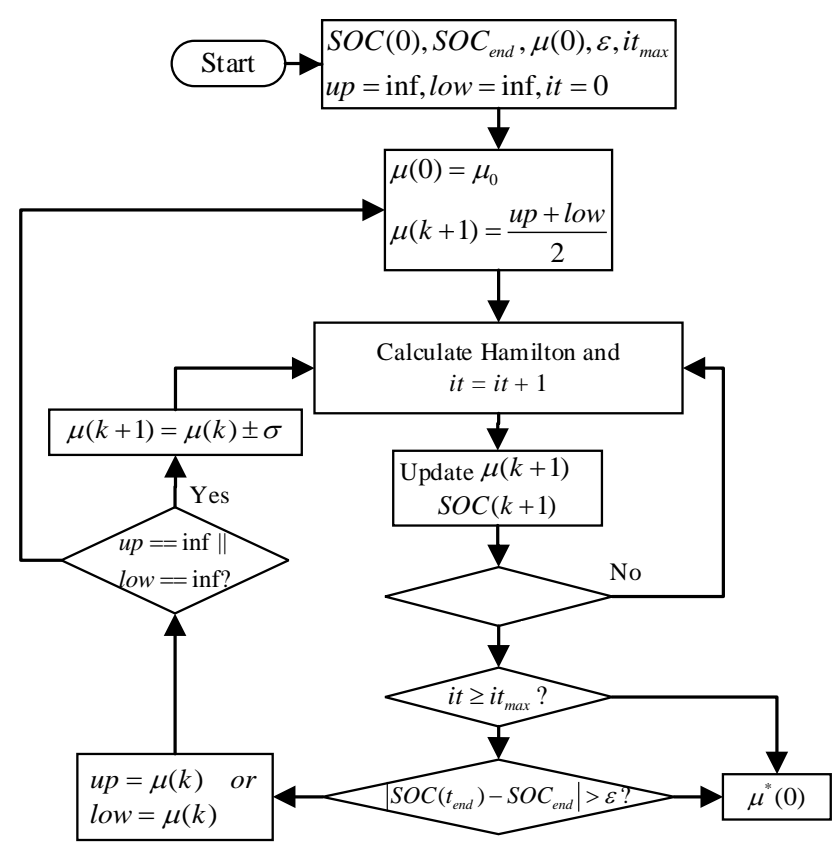

Fig. 10. The flowchart of PMP calculation.

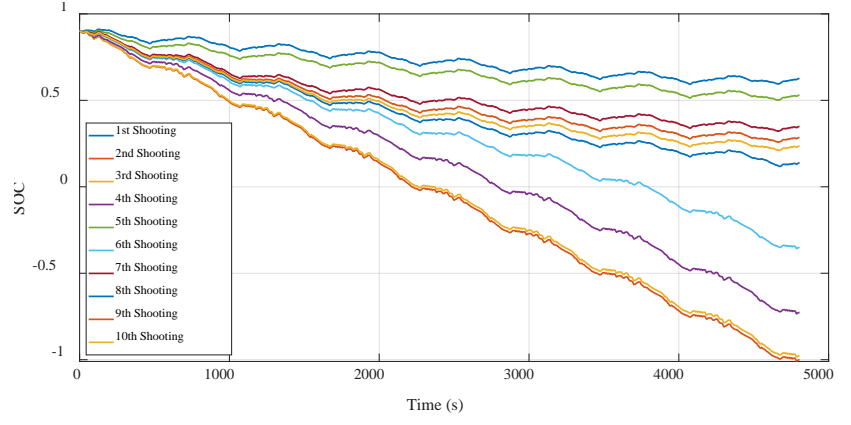

(a)

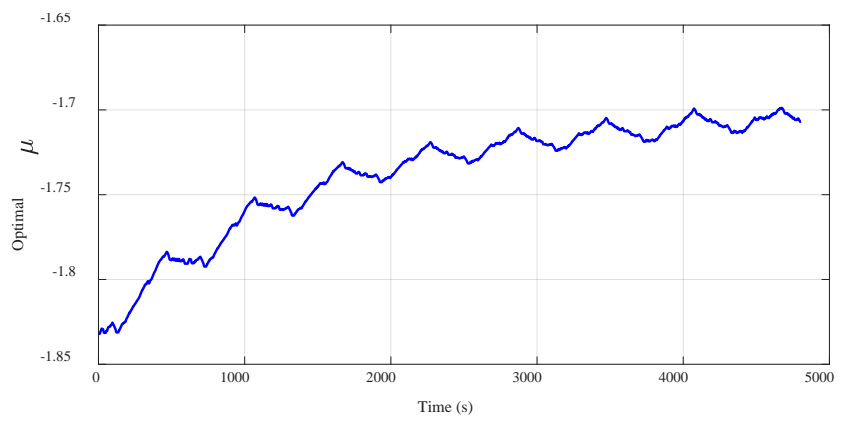

(b)

Fig. 11. The PMP result. (a) illustrates the shooting process and (b) shows the co-state trajectory corresponding to the optimum.

\section{E. Co-State Estimation Based on Recurrent Neural Network}

RNN is a kind of layer-recurrence NN focusing on dealing with time-series prediction problems, and it has been widely adopted in natural language processing [38]. The most important characteristics of RNN is that the neurons in the hidden layer, as shown in Fig. 12, are capable of memorizing the historical information. As can be observed, similar with general feedforward two-layer NNs, the RNN is composed of an input layer, a hidden layer and an output layer. The recurrence weight $W_{h h}$, connecting the hidden output at $t-1$ to the hidden input at $t$, takes charge of delivering historical input to future results. In other words, the network forms a feedback from output to the hidden input, which can be expressed as:

$$
h_{i n}(t)=W_{i h} u(t)+b
$$


where $h_{i n}$ denotes the hidden layer input, $W_{i h}$ denotes the weight from input layer to hidden layer, $u$ denotes the network input, and $b$ represents the bias of input layer. The active function governs the nonlinear mapping from inputs to outputs in the hidden layer, where the tanh function is employed, as:

$$
\tanh \left(\tau_{t}\right)=\frac{e^{\tau_{t}}-e^{-\tau_{t}}}{e^{\tau_{t}}+e^{-\tau_{t}}}=\frac{2}{1+e^{-2 \tau_{t}}}-1
$$

The hidden output at $t$ can be calculated, as:

$$
h(t)=\tanh \left(W_{h h} h(t-1)+h_{i n}(t)\right)
$$

Consequently, the output of network at $t$ can be obtained, as:

$$
o(t)=\tanh \left(W_{h o} h(t)+c\right)
$$

where $W_{\text {ho }}$ denotes the weight from the hidden layer to the output layer, and $c$ is the bias of output layer. From (15), (17) and (18), it can be noticed that $o(t)$, denoted by $f$, is a function of $h(t)$, thus the network output in a unfolded shape can be attained, as:

$$
\begin{aligned}
o(t) & =f(h(t)) \\
& =f(h(t-1), u(t)) \\
& =f(h(t-2), u(t-1), u(t)) \\
& =f(h(0), u(1), u(2), \ldots, u(t))
\end{aligned}
$$

Thanks to the recurrent layer, the output of RNN at any time always relates to all the inputs before the current moment, as clearly illustrated in (19); and therefore, RNN can perform memorial actions in time domain for the past trip information. Furthermore, after the network is well trained, $W_{h h}$ is kept unchanged, implying that the internal recurrence relationship, i.e., the dynamic performance, remains consistent all the time. On this account, by treating the co-state as a time-series signal, the differential relationship of (12) can be approximated by the RNN, thus the co-state can be predicted over the historical RNN inputs consequently. For ensuring the estimation precision, a two-layer RNN with three hidden neurons, called Co-RNN, is introduced in this study to learn the optimal co-state derived from the PMP’s solution. The input variable, represented by $u(\cdot)$ in Fig. 12 for training include the battery SOC, last battery power, engine ratio and percentage of remaining trip length, and the training target is the offline co-state. The results derived by PMP based on the CYCLE505, HWFET, IM240, JC08, LA92, NEDC and REP05 cycles with different repetitions are considered as the training data set, and the results of other cycles are regarded as the validation data set. The proposed Co-RNN is conducted by 
the Elman NN with 4 nodes in the input layer, 3 nodes in the hidden layer and 1 node in the output layer, and the feedback delay of hidden layer is set to 1 step after the output. Note that the purpose of proposed Co-RNN is to provide each time step with an offline optimal initial co-state value, and the necessary co-state correction is also involved due to the estimation error in online operation. At each time step ( $1 \mathrm{~s}$ in this paper), the battery SOC, last battery power value, engine ratio and percentage of remaining trip length are sampled/ calculated first and organized as the Co-RNN input. Then, the RNN calculates its output, i.e., the initial co-state value at that time point, and sends the value into MPC calculation. In the next step, RNN will refresh its hidden layer feedback and repeat the above process until the end of the trip, thereby achieving the co-state regulation.

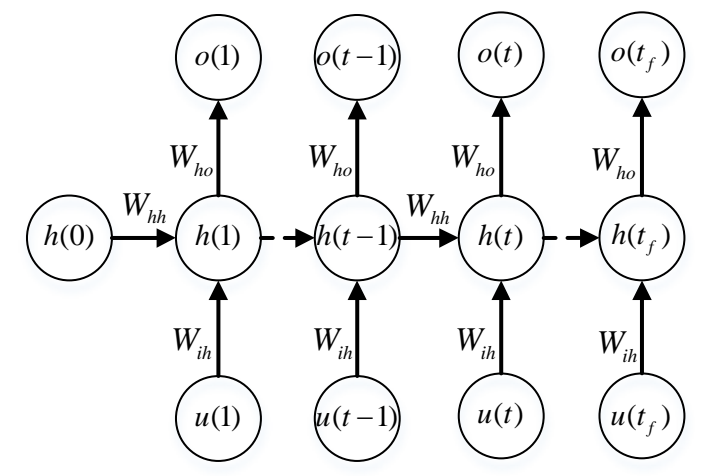

Fig. 12. The structure of RNN.

\section{F. Velocity Prediction Based on RBFNN}

Before applying the MPC, the speed prediction is indispensable to be tackled to supply the future driving information. To provide a short-term speed prediction according to the past vehicle speed, the RBFNN is proposed to serve as the velocity predictor. In a rolling time window, the speed of past $20 \mathrm{~s}$ are imported into the network input, and the network generates five outputs to predict the speed in next $5 \mathrm{~s}$ [39]. By comparison, the radial basis function is finally employed as the neuron kernel in the hidden layer, as:

$$
\varphi\left(\tau_{r}-\rho\right)=\exp \left(-\frac{\left\|\tau_{r}-\rho\right\|^{2}}{2 \sigma^{2}}\right)
$$

where $\varphi$ denotes the RBF kernel, $\rho$ expresses the kernel central point, and $\sigma^{2}$ represents the variance reflecting the kernel variation range. Moreover, seven speed profiles including the CYCLE505, HWFET, IM240, JC08, LA92, NEDC and REP05 are combined to construct the training set, and the remaining seven cycles composite the validation set. The velocity prediction network is established in Matlab/Simulink, and the spread of radial basis functions of the RBFNN is set to 1000 in this study. Note that the predicted speed is assumed as zero in the beginning $20 \mathrm{~s}$ due to incomplete historical information. 


\section{G. Online Implementation}

After establishing the three predictive NNs, the online implementation is elaborated, and the receding horizon optimization is conducted in this part. Due to the compatibility with different optimization algorithms and the capability in dealing with state and control constraints, MPC becomes the most popular online controller in real-time nonlinear optimization fields [40]. The state predictor in MPC contributes to the future variation trend of state variable, and in this study, the vehicle speed is predicted to calculate the SOC variation. The optimizations should be implemented among the prediction horizon for each control period. To ensure the calculation accuracy, the prediction horizon and control domain are both set to $5 \mathrm{~s}$.

Given the current SOC and the reference SOC trajectory, local PMP in the prediction time domain can be solved according to Fig. 10. However, the repetitive iterations for finding the local optimal initial co-state lead to intensive computation burden once given an improper initial co-state value and an exact SOC terminal constraint in each prediction horizon. Thanks to the co-state prediction, the proposed Co-RNN ensures a rough but near-optimal initial co-state, which is beneficial for saving the calculation time to some extent. In this case, the linear SOC reference trajectories regarding the travel distance in the global trip and local prediction are combined together to obtain the final reference value, as:

$$
\left\{\begin{array}{l}
x_{r e f}^{l}(t+k)=x(t)-\frac{\left(x(0)-x_{t_{f}}\right) \sum_{k=1}^{N} v_{p}(t+k)}{L_{\text {trip }}-L(t)} \\
x_{\text {ref }}^{g}(t+k)=x_{t_{f}}+\frac{\left(x(0)-x_{t_{f}}\right)\left(L_{\text {trip }}-L(t)-\sum_{k=1}^{N} v_{p}(t+k)\right)}{L_{\text {trip }}}
\end{array}\right.
$$

where $x_{r e f}(t+k)$ represents the SOC reference value in the $k$ th prediction at $t$, and the superscripts $g$ and $l$ denotes the global reference and local reference, respectively; $L_{\text {trip }}$ represents the total trip distance, $L(t)$ is the traveled distance at $t, N$ expresses the predicting length, and $v_{p}$ denotes the predicted velocity. The global reference refers to the SOC trajectory that starts from the initial SOC in the beginning of trip and ends at the desired terminal value when the trip ends, reflecting the overall SOC variation trend in the whole trip; while the local one starts from the current SOC feedback and ends at the desired terminal SOC, revealing the average SOC variation trend in the remaining trip. The main purpose is to track the global SOC trajectory with the local regulation. These two trajectories will merge together if the current SOC, as expected, locates exactly on the 
global reference curve. Generally, the two trajectories will form a gradually diminished region if the current SOC is out of the global trajectory, thus necessitating the feedback algorithm to regulate the SOC to approximate the global one. It deserves to be declared that the proposed SOC reference in (21) is an near-optimal value for unknown driving conditions, thus excessively precise track of reference is worthless for online application. To track the reference trajectory under loose conditions, the SOC tracking tolerance is magnified according to the both reference value, as:

$$
\left\{\begin{array}{lll}
\bar{X}_{\text {lim }}(t)=x_{\text {ref }}^{g}(t) & \underline{x}_{\text {lim }}(t)=x_{\text {ref }}^{l}(t), & x_{\text {ref }}^{g}(t)>x_{\text {ref }}^{l}(t) \\
\bar{x}_{\text {lim }}(t)=x_{\text {ref }}^{l}(t) & \underline{x}_{\text {lim }}(t)=x_{\text {ref }}^{g}(t), & x_{\text {ref }}^{g}(t) \leq x_{\text {ref }}^{l}(t)
\end{array}\right.
$$

where $\bar{x}_{\text {lim }}$ and $\underline{x}_{\text {lim }}$ represent the upper and lower constraints of SOC, respectively. In online implementation, the co-state provided by the Co-NN at each step is a near-optimal co-state value, rather than an accurate value, thanks to the prediction error and driving uncertainty in the prediction horizon. When the near-optimal value of co-state is directly applied in the Hamilton function in (10) without correction, the accumulative deviation in the whole cycle might result in rude SOC tracking failure. To guarantee that the SOC terminates within the constraint tolerance in the end of predicted horizon, the co-state correction is conducted according to the predicted SOC and reference value at the end of prediction domain, as:

$$
\left\{\begin{array}{l}
e=x_{r e f}^{g}(N)-x(N) \\
\psi(e)=K \cdot e /(|e|+o) \\
\mu^{*}=\mu+\psi(e)
\end{array}\right.
$$

where $e$ denotes the SOC error at the prediction terminal, and $\psi$ represents a softmax function to avoid an exaggerated adjustment. The calculation procedure of rolling horizon optimization is depicted in Fig. 13. At each time step, the necessary feedback sampling and trip information, such as the battery SOC, velocity, trip distance, are collected first by the controller. Afterwards, the battery SOC, vehicle speed, demanded power and engine ratio are sent to the M-NN, and the predicted vehicle operating mode are calculated. The battery SOC, previous battery power, engine ratio and percentage of remaining trip length are sent to the Co-NN to attain the initial co-state; the speed of past $20 \mathrm{~s}$ is acquired and sent to the V-NN to predict the speed of future $5 \mathrm{~s}$; moreover, the SOC reference regarding (21) and (22) will be calculated. Given all the NN output, the battery power is calculated by solving the Hamilton function with the constant co-state for every second in the MPC module; and meanwhile, the SOC variation in the same time domain is predicted to judge if the SOC violates 
the reference boundaries. If so, the co-state value will be corrected according to (23), and then the processes of battery power SOC update and co-state correction will be conducted repeatedly until the final state converges within the boundaries. Finally, with the well tracked SOC trajectory, the battery power in the first second of MPC domain along with the operating mode commands from M-NN will be sent to the local controllers to achieve the energy distribution. Note that a protection mechanism is introduced to avoid infinite iteration; that said, if the final state is not within SOC boundaries, and the two adjacent co-state modifications simultaneously result in the boundary power and the corresponding SOC error variation in the same direction, i.e., $e(k) \times e(k-1) \geq 0$, the boundary power should be directly the output.

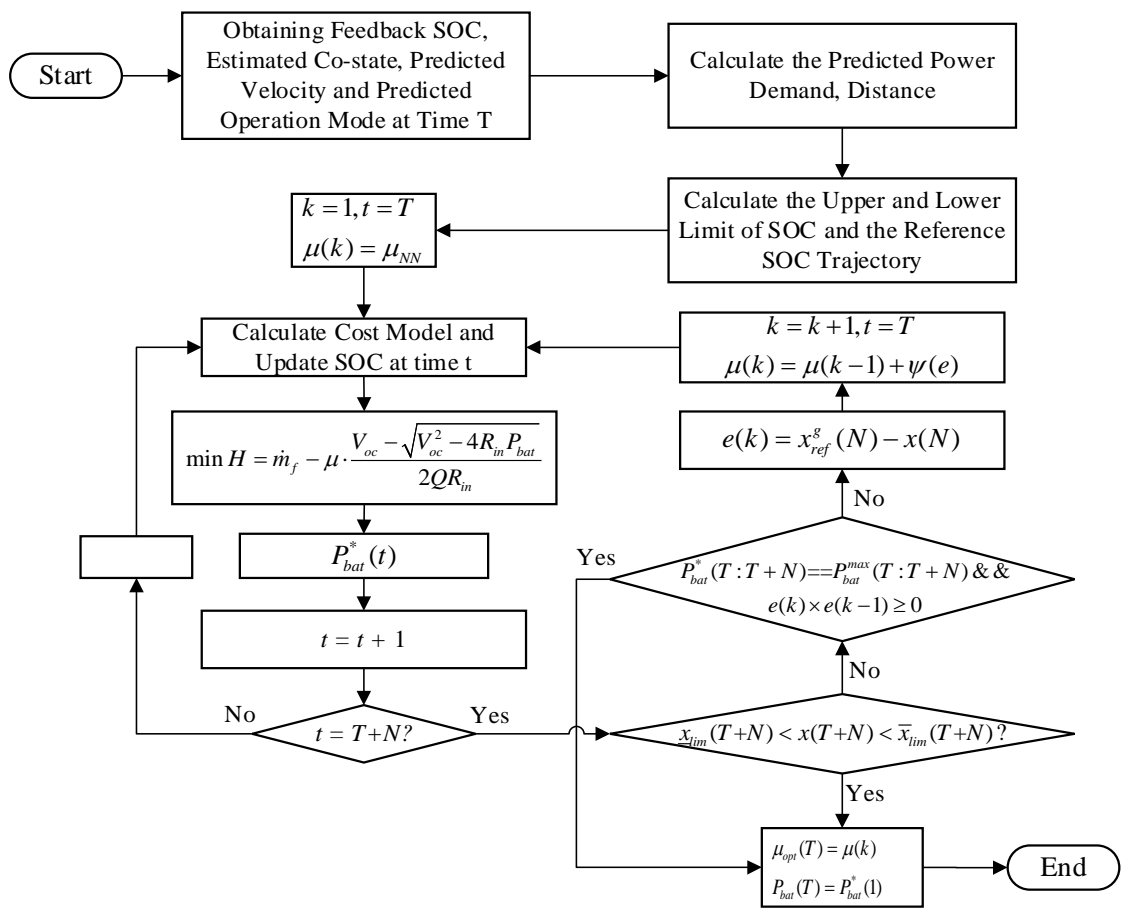

Fig. 13. The online calculation procedure. $k$ denotes the iteration times for initial co-state modification, $t$ and $T$ represent the prediction time and current time, respectively, and $\mu_{N N}$ indicates the output of Co-NN at $T$.

\section{RESULTS AND DISCUSSION}

In this section, the simulations were conducted to validate the feasibility of proposed method. The driving cycles including SC03, UDDS, US06, WLTC, WVUS, KM1 and KM2 with different repetitions are considered as the validating driving condition. First, to validate the effectiveness and performance of the proposed NNs, the results and analysis of M-NN, Co-NN and V-NN are presented and discussed with respect to the mode prediction, co-state prediction and future speed prediction, respectively. Secondly, the energy management 
effect by the proposed EMS are demonstrated, and further comparisons with other widely employed approaches are discussed in detail as well.

\section{A. Mode Prediction Result Analysis}

The prediction result of proposed M-NN under the selected driving cycles is shown in Table III. The satisfactory accuracy of $99.08 \%$ manifests the efficacy of mode recognition by the proposed M-NN algorithm. In our previous work, only the battery SOC, vehicle speed and demanded power are exerted to train the mode prediction network [41]. For the sake of illustrating the impact of different input parameters, three groups of parameters, namely CG1, CG2 and CG3, are selected for the M-NN training. Thereinto, CG1 contains the vehicle velocity, power demand and battery SOC; CG2 includes the vehicle velocity, power demand and engine ratio; and CG3 is the preferred method proposed in Section III, and involves the vehicle velocity, power demand, battery SOC and engine ratio. The recognition results of M-NNs trained by three different groups are listed in Table III, from which we find that the prediction accuracy increases when considering the engine ratio, and the proposed method outperforms other groups of parameter set. Meanwhile, the result implies the importance and effectiveness of the feature of engine ratio in mode prediction.

Table III. M-NN output comparison for different training groups.

\begin{tabular}{|c|c|c|c|c|c|c|c|}
\hline \multirow{2}{*}{$\begin{array}{l}\text { Group } \\
\text { Name }\end{array}$} & \multicolumn{4}{|c|}{ Character parameter Name } & \multirow{2}{*}{$\begin{array}{l}\text { Times of Correct } \\
\text { Prediction }\end{array}$} & \multirow{2}{*}{$\begin{array}{l}\text { Times of Incorrect } \\
\text { Prediction }\end{array}$} & \multirow[t]{2}{*}{ Accuracy } \\
\hline & SOC & Engine Ratio & Vehicle Speed & Power Demand & & & \\
\hline CG1 & Yes & No & Yes & Yes & 200338 & 6950 & $96.65 \%$ \\
\hline CG2 & No & Yes & Yes & Yes & 205042 & 2246 & $98.92 \%$ \\
\hline CG3 & Yes & Yes & Yes & Yes & 205380 & 1908 & $99.08 \%$ \\
\hline
\end{tabular}

B. Co-State Prediction Result Analysis

The predictive performance of Co-NN is depicted in Fig. 14. To quantify the evaluation, the goodness of fit (GOF) that integrally reflects fitting quality on curve shape and error is introduced, as:

$$
R^{2}=1-\frac{\sum_{k=0}^{N}\left(y_{k}-\hat{y}_{k}\right)^{2}}{\sum_{k=0}^{N}\left(y_{k}-\bar{y}\right)^{2}}
$$

where $R^{2}$ represents the GOF, $y_{k}$ denotes the optimal co-state at the $k$ th sampling step, $\bar{y}$ express the sample average value of optimal co-state, and $\hat{y}_{k}$ indicates the output of Co-RNN at the $k$ th step. In this case, the data set implemented in the mode prediction training is also involved for co-state prediction training, and the remaining data is employed for validation. 

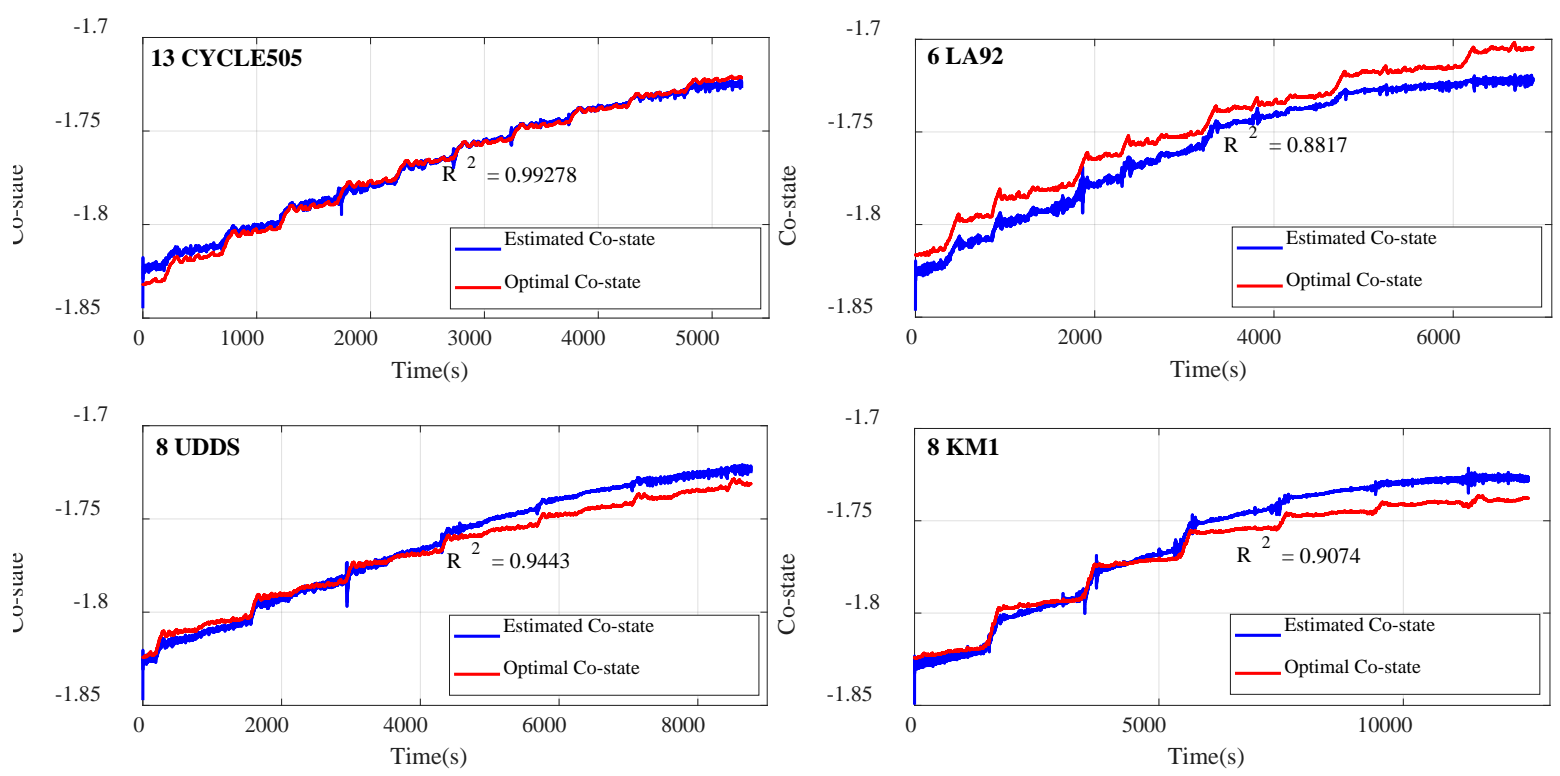

Fig. 14. Co-RNN prediction results.

As can be seen from Fig. 14, the output of Co-RNN can effectively follow the dynamic variation of costate in the aspects of variation trend and curve shape. All the $R^{2}$ is more than 0.88 , proving its preferable approximation performance. Despite slight estimation error at the end of the cycle, the predicted co-state by the Co-RNN can precisely track the optimal values, thereby providing the initial value for iteration at each time step. To say the least, even if the prediction error exists, the near-optimal co-state can be iteratively corrected in the MPC framework according to the SOC feedback and its reference value to ensure the optimality of real-time solution. Compared with adopting a random or constant initial co-state value, the proposed method leads to shorter iteration time, as illustrated in Table V. Furthermore, by comparing with the co-state estimation results obtained by the back propagation NN (BPNN) in [42], the estimation results by Co-RNN show less fluctuation and are closer to the optimum, proving its preferable regulation performance when predicting the co-state.

\section{Velocity Prediction Result Analysis}

In [27], the BPNN implemented for velocity prediction is proved feasible and hence is treated as an alternative for comparison. The speed prediction results of RBFNN and BPNN under the WLTC are shown in Fig. 15, and the overall prediction root mean square error (RMSE) of cycles in the validation set are presented in Table IV. A common phenomenon of two predictors lies in that sharper speed variation generally results in larger prediction error, while the plateau on the speed profile always performs better prediction accuracy. With respect to the prediction accuracy, both the RBFNN and BPNN show the similar prediction performance; however, the difference is that the RBFNN's prediction is more stable at higher speed; and in contrast more frequent fluctuation occurs in the BPNN's prediction result. According to the vehicle dynamics, the speed error 
can incur cubic deviated error of demanded power [43], thus further deteriorating the power distribution accuracy. Obviously, the RBFNN indicates better performance in high speed prediction. From the overall point of view, the RMSE listed in Table IV proves the adaptivity of RBFNN for most of the driving conditions.
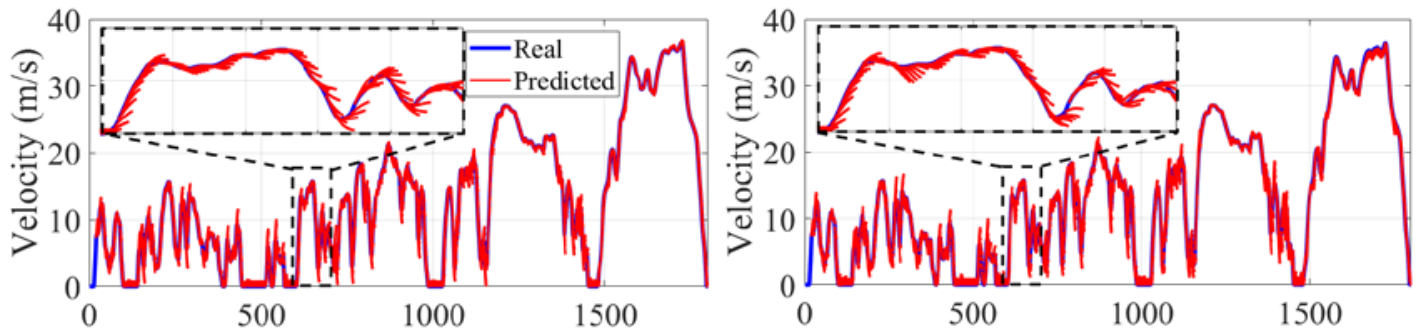

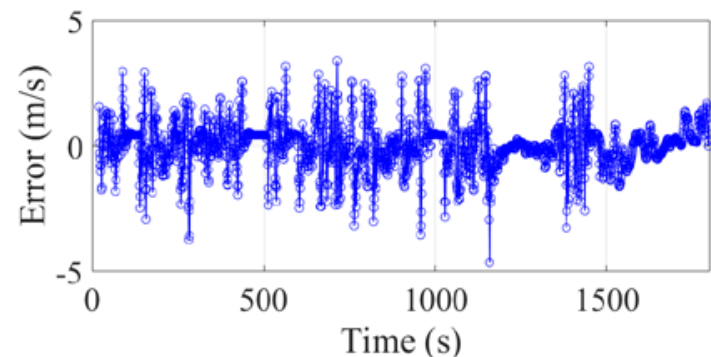

(a)

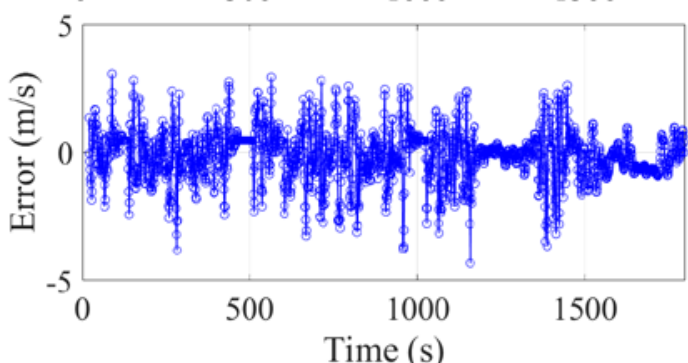

(b)

Fig. 15. Velocity prediction result: (a) Result of RBFNN; (b) Result of BPNN.

Table IV. RMSE comparison of predicted speed.

\begin{tabular}{llllllll}
\hline Cycle Name & \multicolumn{1}{c}{ SC03 } & UDDS & US06 & WLTC & WVUSUB & KM1 & KM2 \\
\hline \multirow{2}{*}{ RBFNN } & 1.46 & 1.11 & 1.93 & 1.12 & 0.94 & 1.38 & 1.16 \\
& $(\uparrow 12.57 \%)$ & $(\uparrow 11.20 \%)$ & $(\downarrow 14.88 \%)$ & $(\uparrow 4.27 \%)$ & $(\uparrow 18.97 \%)$ & $(\uparrow 19.30 \%)$ & $(\uparrow 37.30 \%)$ \\
BPNN & 1.67 & 1.25 & 1.68 & 1.17 & 1.16 & 1.71 & 1.85 \\
\hline
\end{tabular}

D. Energy Management Validation and Results Comparison

The performance of proposed method is compared with the result derived from the widely adopted CD/CS strategy. By implementing the CD/CS scheme, the controller priorly makes full use of the stored electricity to propel the vehicle until the battery SOC reaches the low threshold (0.294 in this study), then the controller attempts to turn on the ICE to sustain the SOC around the low value. Fig. 16 shows the comparison of SOC and cost for the proposed strategy and CD/CS under five WLTC cycles, respectively; and the corresponding DP results are also presented to further evaluate the performance of proposed algorithm. The SOC results indicate that the proposed NN-MPC strategy can approximate the optimal solution, and with the help of feedback correction, the terminal SOC can always satisfy the optimal DOD condition. As shown in Fig. 16 (b), the final cost of NN-MPC, CD/CS and DP is \$3.31, \$3.56 and \$2.80, respectively; and the savings of proposed strategy and DP are respectively $7.43 \%$ and $21.35 \%$, compared with the CD/CS scheme; and the proposed method can be evaluated to reaches $34.80 \%$ savings of DP, if CD/CS and DP are employed as the lower and higher benchmark boundaries, respectively. Fig. 17 shows the corresponding engine operating points of the CD/CS 
and proposed strategy, respectively. The distribution of engine operating points explicitly reveals that, by the $\mathrm{CD} / \mathrm{CS}$ strategy, the engine frequently starts up and works in the low power and low efficiency region, thereby resulting in higher cost. By contrast, the proposed algorithm always facilitates the engine to operate in the higher power and higher efficiency region, demonstrating lower on/off frequency of engine and higher fuel economy.

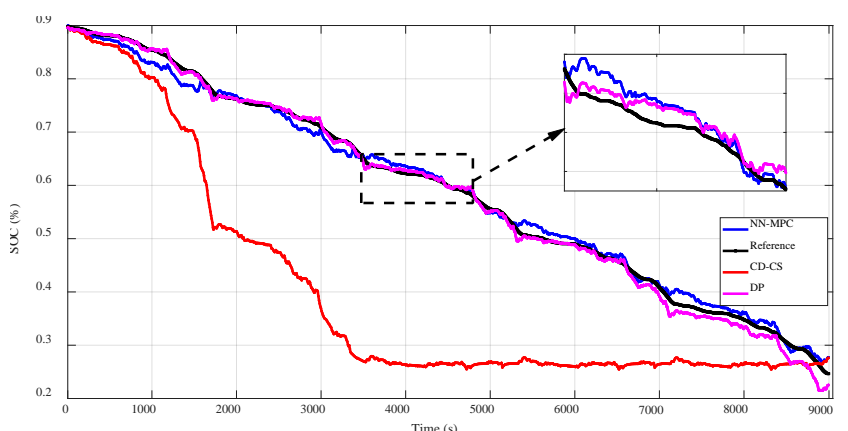

(a)

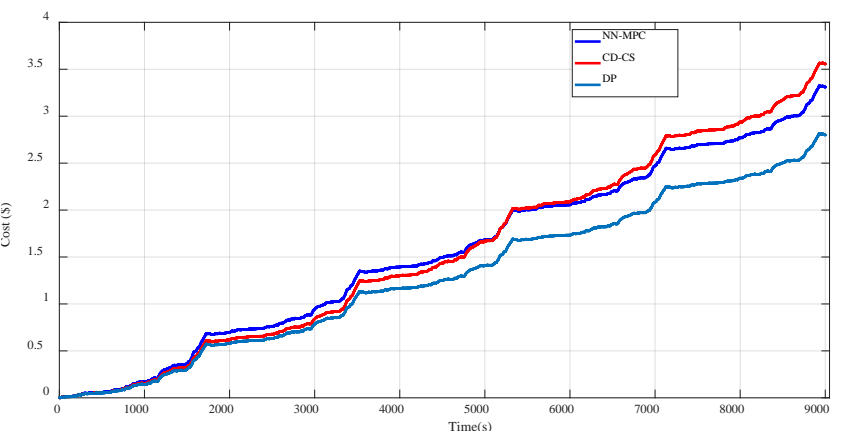

(b)

Fig. 16. Simulation result comparison.

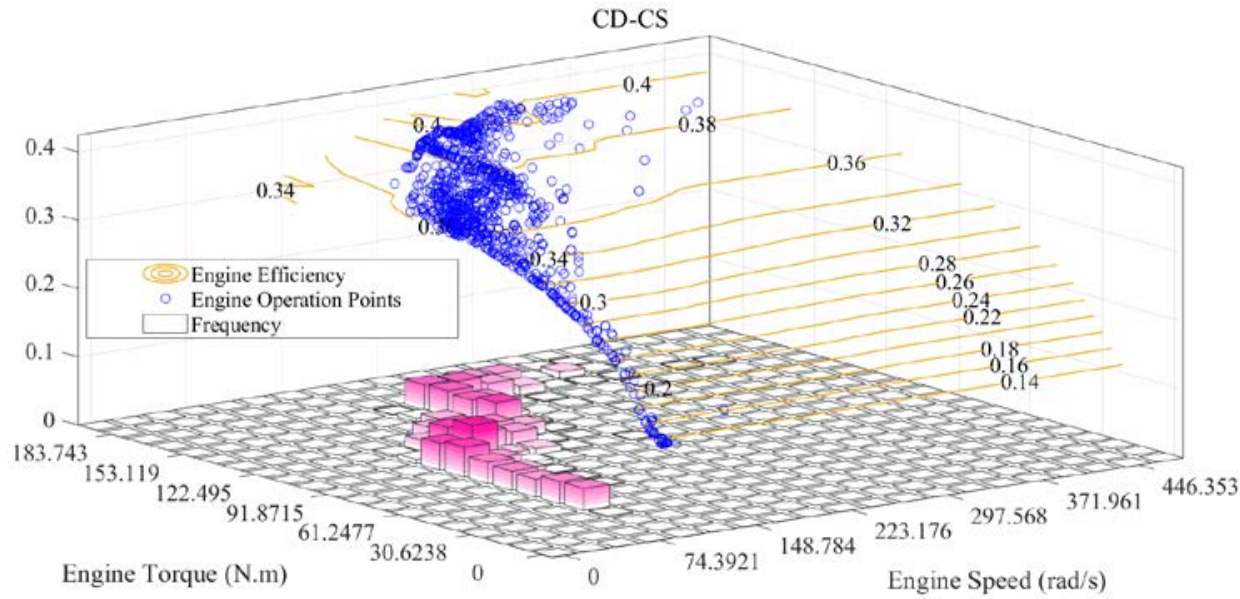

(a)

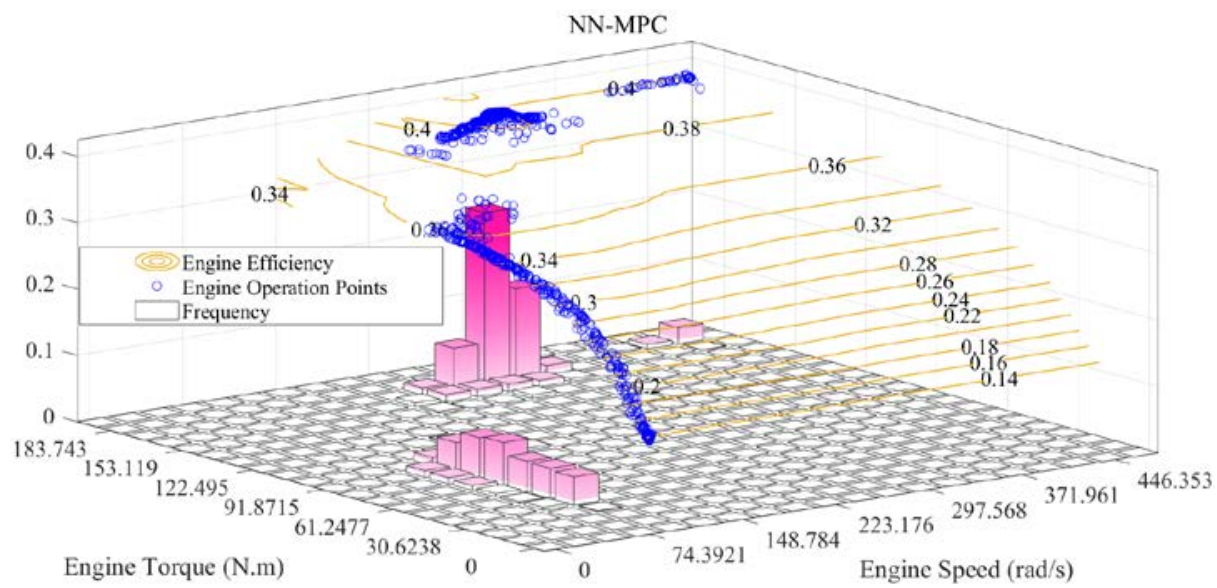

(b)

Fig. 17. The engine operating point and the corresponding frequency.

To further validate the effectiveness and adaptiveness of proposed algorithm, the ECMS and PMP-based MPC (PMP-MPC) strategy are also employed for comparison. The PMP-MPC refers to the same framework 
with the proposed strategy. The only difference lies in that the proposed algorithm applies the multi NNs to achieve the local optimization, in contrast to the PMP applied in the PMP-MPC algorithm for local optimization. For fair comparison, the equivalent factor of ECMS is tuned as close as possible to meet the requirement of the same terminal SOC for every cycle; as to the PMP-MPC algorithm, the reference SOC trajectory is the same with that in NN-MPC algorithm, and the initial value of co-state at each time step remains the same. The comprehensive results of the noticed methods, along with the global DP and PMP as the benchmarks, are listed in Table V.

Table V. Simulation results comparison.

\begin{tabular}{llllllllll}
\hline $\begin{array}{l}\text { Cycle } \\
\text { Name }\end{array}$ & Method & $\begin{array}{l}\text { End } \\
\text { SOC }\end{array}$ & $\begin{array}{l}\text { Total } \\
\text { Cost } \\
(\$)\end{array}$ & $\begin{array}{l}\text { Periodic } \\
\text { Time }(\mathrm{s})\end{array}$ & Cycle Name & Method & $\begin{array}{l}\text { End } \\
\text { SOC }\end{array}$ & $\begin{array}{l}\text { Total } \\
\text { Cost } \\
(\$)\end{array}$ & $\begin{array}{l}\text { Periodic } \\
\text { Time (s) }\end{array}$ \\
\hline \multirow{6}{*}{ 13 SC03 } & ECMS & 0.264 & 2.22 & 0.0041 & & ECMS & 0.251 & 2.95 & 0.0052 \\
& Proposed & 0.265 & 1.73 & 0.0109 & & Proposed & 0.214 & 2.00 & 0.0108 \\
& PMP-MPC & 0.264 & 1.76 & 0.0202 & \multirow{2}{*}{ 8 WVUSUB } & PMP-MPC & 0.259 & 2.07 & 0.0141 \\
& DP & 0.266 & 1.38 & - & & DP & 0.226 & 1.68 & - \\
& PMP & 0.267 & 1.31 & - & & PMP & 0.237 & 1.56 & - \\
\hline \multirow{5}{*}{6 UDDS } & ECMS & 0.277 & 2.14 & 0.0047 & & ECMS & 0.234 & 3.00 & 0.0041 \\
& Proposed & 0.263 & 1.56 & 0.0102 & & Proposed & 0.243 & 2.32 & 0.0101 \\
& PMP-MPC & 0.265 & 1.58 & 0.0181 & 6 KM1 & PMP-MPC & 0.265 & 2.37 & 0.0225 \\
& DP & 0.252 & 1.28 & - & & DP & 0.237 & 1.89 & - \\
& PMP & 0.257 & 1.23 & - & & PMP & 0.230 & 1.87 & - \\
\hline \multirow{5}{*}{5 WLTC } & ECMS & 0.278 & 3.77 & 0.0043 & & ECMS & 0.254 & 2.97 & 0.0047 \\
& Proposed & 0.279 & 3.31 & 0.0117 & & Proposed & 0.261 & 1.94 & 0.0108 \\
& PMP-MPC & 0.278 & 3.41 & 0.0180 & 13 KM2 & PMP-MPC & 0.262 & 1.98 & 0.0253 \\
& DP & 0.233 & 2.80 & - & & DP & 0.258 & 1.57 & - \\
& PMP & 0.231 & 2.46 & - & & PMP & 0.258 & 1.49 & - \\
\hline
\end{tabular}

As can be found, by adopting the maximum cost of each cycle, attained by ECMS, as the cost baseline, the savings of proposed algorithm are 22.07\%, 27.10\%, 12.2\%, 32.20\%, 22.67\% and 34.68\% for 13 SC03, 6 UDDS, 5 WLTC, 8 WVUSUB, 6 KM1 and 13 KM2 cycles, respectively, and the corresponding exceeding costs are respectively $25.36 \%, 21.88 \%, 18.21 \%, 19.05 \%, 22.75 \%$ and 23.57\%, compared with those by DP. Except for DP and PMP, the proposed algorithm always shows better performance in total cost under different driving cycles. The reason is that the algorithm not only carries out a local optimum with respect to reference constraints, but also employs the optimal information from NNs trained by DP and PMP to attain the future driving knowledge a priori, which is inaccessible in other two online approaches. Given the nearly same terminal SOC for each driving cycle, the total cost can be merely regarded as the fuel cost; that is to say, with the determined 
optimal DOD, the total cost is strongly related to a better regulated engine operation, which is also verified in Fig. 17. As to the calculation time, the ECMS has the shortest computational load within $5.2 \mathrm{~ms}$ but with the most total cost due to its simplest implementation. Comparing with the PMP-MPC, the time savings of 23.40\% to $57.31 \%$ are reached under the $13 \mathrm{KM} 2$ cycle in Table $\mathrm{V}$ by providing a near-optimal co-state online to accelerate the iteration, and the slacked SOC boundaries also contribute to unnecessary SOC traction effort, thus incurring less computational load but better fuel economy.

\section{CONCLUSIONS}

In this study, a neural network enhanced online energy management strategy is developed based on model predictive control. Firstly, the structure of concerned multi-mode plug-in hybrid electric vehicle is investigated in detail, then dynamic programming is employed to find the global optimal control for all the referred driving cycles. Sequentially, the mode recognition neural network is introduced and trained for online mode prediction. To cope with difficulties in batter power modeling, Pontryagin's minimum principle is leveraged to find the optimal co-state sequence, by which the recurrent neural network is trained to provide real-time co-state value acting as the initial value for battery power optimization. Finally, the speed prediction is conducted by the radial basis function neural network, and the online model predictive control algorithm is carried out, with the improved state of charge reference trajectory and its slacked boundaries, for solving the battery power command. The simulation results manifest that the proposed method achieves the cost savings by $12.2 \%$ to $34.68 \%$, compared with equivalent consumption minimum strategy, and attains the similar total cost but with maximum 57.31\% calculation labor reduction, compared with Pontryagin’s minimum principle-based model predictive control, thereby demonstrating satisfactory online eco-driving performance.

To further explore the economic potential of plug-in hybrid electric vehicles, battery degradation needs be considered, and the self-learning-based energy management strategies for customized vehicles and drivers will be carefully investigated in our future research.

\section{ACKNOWLEDGEMENTS}

This work was supported in part by the National Natural Science Foundation of China (No. 61763021 and No. 51775063), in part by the National Key R\&D Program of China (No. 2018YFB0104900), and in part by 
the EU-funded Marie Skłodowska-Curie Individual Fellowships Project under Grant 845102-HOEMEV-

H2020-MSCA-IF-2018.

\section{REFERENCES}

[1] Y. Zhou, A. Ravey, and M.-C. Péra, "A survey on driving prediction techniques for predictive energy management of plug-in hybrid electric vehicles," Journal of Power Sources, vol. 412, pp. 480-495, 2019, doi: 10.1016/j.jpowsour.2018.11.085.

[2] Z. Chen, X. Shu, R. Xiao, W. Yan, Y. Liu, and J. Shen, "Optimal charging strategy design for lithium - ion batteries considering minimization of temperature rise and energy loss," International Journal of Energy Research, vol. 43, no. 9, pp. 4344-4358, 2019.

[3] M. A. Hannan, M. M. Hoque, A. Mohamed, and A. Ayob, "Review of energy storage systems for electric vehicle applications: Issues and challenges," Renewable and Sustainable Energy Reviews, vol. 69, pp. 771-789, 2017, doi: 10.1016/j.rser.2016.11.171.

[4] K. Song, F. Li, X. Hu, L. He, W. Niu, S. Lu, and T. Zhang,, "Multi-mode energy management strategy for fuel cell electric vehicles based on driving pattern identification using learning vector quantization neural network algorithm," Journal of Power Sources, vol. 389, pp. 230-239, 2018, doi: 10.1016/j.jpowsour.2018.04.024.

[5] A. Rezaei, J. B. Burl, B. Zhou, and M. Rezaei, "A New Real-Time Optimal Energy Management Strategy for Parallel Hybrid Electric Vehicles," IEEE Transactions on Control Systems Technology, vol. 27, no. 2, pp. 830837, 2019, doi: 10.1109/tcst.2017.2775184.

[6] M. Li, H. He, M. Yan, and J. Peng, "Variable horizon MPC for energy management on dual planetary gear hybrid electric vehicle," Energy Procedia, vol. 152, pp. 636-642, 2018, doi: 10.1016/j.egypro.2018.09.223.

[7] J. Wu, J. Ruan, N. Zhang, and P. D. Walker, "An Optimized Real-Time Energy Management Strategy for the Power-Split Hybrid Electric Vehicles," IEEE Transactions on Control Systems Technology, vol. 27, no. 3, pp. 1194-1202, 2019, doi: 10.1109/tcst.2018.2796551.

[8] D. Zhou, A. Al-Durra, I. Matraji, A. Ravey, and F. Gao, "Online Energy Management Strategy of Fuel Cell Hybrid Electric Vehicles: A Fractional-Order Extremum Seeking Method," IEEE Transactions on Industrial Electronics, vol. 65, no. 8, pp. 6787-6799, 2018, doi: 10.1109/tie.2018.2803723.

[9] L. Zhang, W. Liu, and B. Qi, "Innovation design and optimization management of a new drive system for plug-in hybrid electric vehicles," Energy, vol. 186, 2019, doi: 10.1016/j.energy.2019.07.153.

[10] J. Wang, J. Wang, Q. Wang, and X. Zeng, "Control rules extraction and parameters optimization of energy management for bus series-parallel AMT hybrid powertrain," Journal of the Franklin Institute, vol. 355, no. 5, pp. 2283-2312, 2018, doi: 10.1016/j.jfranklin.2017.12.039.

[11] M. Yan, M. Li, H. He, J. Peng, and C. Sun, "Rule-based energy management for dual-source electric buses extracted by wavelet transform," Journal of Cleaner Production, vol. 189, pp. 116-127, 2018, doi: 10.1016/j.jclepro.2018.04.054.

[12] Y. Liu, J. Gao, D. Qin, Y. Zhang, and Z. Lei, "Rule-corrected energy management strategy for hybrid electric vehicles based on operation-mode prediction," Journal of Cleaner Production, vol. 188, pp. 796-806, 2018, doi: 10.1016/j.jclepro.2018.04.024.

[13] J. Peng, H. He, and R. Xiong, "Rule based energy management strategy for a series-parallel plug-in hybrid electric bus optimized by dynamic programming," Applied Energy, vol. 185, pp. 1633-1643, 2017, doi: 10.1016/j.apenergy.2015.12.031.

[14] B. Xu, D. Rathod, D. Zhang, A. Yebi, X. Zhang, X. Li, and Z. Filipi, "Parametric study on reinforcement learning optimized energy management strategy for a hybrid electric vehicle," Applied Energy, vol. 259, 2020, doi: 10.1016/j.apenergy.2019.114200.

[15] R. Schmid, J. Bürger, and N. Bajcinca, "A comparison of PMP-based Energy Management Strategies for Plugin-Hybrid Electric Vehicles," IFAC-PapersOnLine, vol. 52, no. 5, pp. 592-597, 2019, doi: 10.1016/j.ifacol.2019.09.094.

[16] L. Qiu, L. Qian, H. Zomorodi, and P. Pisu, "Global optimal energy management control strategies for connected four-wheel-drive hybrid electric vehicles," IET Intelligent Transport Systems, vol. 11, no. 5, pp. 264-272, 2017, doi: 10.1049/iet-its.2016.0197.

[17] S. Zhang and R. Xiong, "Adaptive energy management of a plug-in hybrid electric vehicle based on driving pattern recognition and dynamic programming," Applied Energy, vol. 155, pp. 68-78, 2015, doi: 10.1016/j.apenergy.2015.06.003.

[18] S. Uebel, N. Murgovski, C. Tempelhahn, and B. Baker, "Optimal Energy Management and Velocity Control of Hybrid Electric Vehicles," IEEE Transactions on Vehicular Technology, vol. 67, no. 1, pp. 327-337, 2018, doi: 10.1109/tvt.2017.2727680. 
[19] X. Lü, Y. Wu, J. Lian, Y. Zhang, C. Chen, P. Wang, and L. Meng, "Energy management of hybrid electric vehicles: A review of energy optimization of fuel cell hybrid power system based on genetic algorithm," Energy Conversion and Management, vol. 205, 2020, doi: 10.1016/j.enconman.2020.112474.

[20] S. Onori and L. Tribioli, "Adaptive Pontryagin’s Minimum Principle supervisory controller design for the plugin hybrid GM Chevrolet Volt," Applied Energy, vol. 147, pp. 224-234, 2015, doi: 10.1016/j.apenergy.2015.01.021. Z. Chen, C. C. Mi, B. Xia, and C. You, "Energy management of power-split plug-in hybrid electric vehicles based on simulated annealing and Pontryagin's minimum principle," Journal of Power Sources, vol. 272, pp. 160-168, 2014, doi: 10.1016/j.jpowsour.2014.08.057.

[22] K. Namwook, C. Sukwon, and P. Huei, "Optimal Control of Hybrid Electric Vehicles Based on Pontryagin's Minimum Principle," IEEE Transactions on Control Systems Technology, vol. 19, no. 5, pp. 1279-1287, 2011, doi: 10.1109/tcst.2010.2061232.

[23] S. Xie, X. Hu, Q. Zhang, X. Lin, B. Mu, and H. Ji, "Aging-aware co-optimization of battery size, depth of discharge, and energy management for plug-in hybrid electric vehicles," Journal of Power Sources, vol. 450, 2020, doi: 10.1016/j.jpowsour.2019.227638.

[24] N. Guo, J. Shen, R. Xiao, W. Yan, and Z. Chen, "Energy management for plug-in hybrid electric vehicles considering optimal engine ON/OFF control and fast state-of-charge trajectory planning," Energy, vol. 163, pp. 457-474, 2018, doi: 10.1016/j.energy.2018.08.116.

[25] J. Yuan and L. Yang, "Predictive energy management strategy for connected 48V hybrid electric vehicles," Energy, vol. 187, 2019, doi: 10.1016/j.energy.2019.115952.

[26] A. Rezaei, J. B. Burl, and B. Zhou, "Estimation of the ECMS Equivalent Factor Bounds for Hybrid Electric Vehicles," IEEE Transactions on Control Systems Technology, vol. 26, no. 6, pp. 2198-2205, 2018, doi: 10.1109/tcst.2017.2740836.

[27] Y. Liu, J. Li, Z. Chen, D. Qin, and Y. Zhang, "Research on a multi-objective hierarchical prediction energy management strategy for range extended fuel cell vehicles," Journal of Power Sources, vol. 429, pp. 55-66, 2019, doi: 10.1016/j.jpowsour.2019.04.118.

[28] S. Xie, X. Hu, S. Qi, X. Tang, K. Lang, Z. Xin, and J. Brighton, "Model predictive energy management for plugin hybrid electric vehicles considering optimal battery depth of discharge," Energy, vol. 173, pp. 667-678, 2019, doi: 10.1016/j.energy.2019.02.074.

[29] Y. Zeng, Y. Cai, G. Kou, W. Gao, and D. Qin, "Energy Management for Plug-In Hybrid Electric Vehicle Based on Adaptive Simplified-ECMS," Sustainability, vol. 10, no. 6, 2018, doi: 10.3390/su10062060.

[30] Y. Li, H. He, A. Khajepour, H. Wang, and J. Peng, "Energy management for a power-split hybrid electric bus via deep reinforcement learning with terrain information," Applied Energy, vol. 255, 2019, doi: 10.1016/j.apenergy.2019.113762.

[31] G. Du, Y. Zou, X. Zhang, Z. Kong, J. Wu, and D. He, "Intelligent energy management for hybrid electric tracked vehicles using online reinforcement learning," Applied Energy, vol. 251, 2019, doi: 10.1016/j.apenergy.2019.113388.

[32] Y. L. Murphey, J. Park, L. Kiliaris, M. L. Kuang, M. A. Masrur, A. M. Phillips, and Q. Wang, "Intelligent Hybrid Vehicle Power Control-Part II: Online Intelligent Energy Management," IEEE Transactions on Vehicular Technology, vol. 62, no. 1, pp. 69-79, 2013, doi: 10.1109/tvt.2012.2217362.

[33] H. Liu, X. Li, W. Wang, L. Han, and C. Xiang, "Markov velocity predictor and radial basis function neural network-based real-time energy management strategy for plug-in hybrid electric vehicles," Energy, vol. 152, pp. 427-444, 2018, doi: 10.1016/j.energy.2018.03.148.

[34] H. Tian, Z. Lu, X. Wang, X. Zhang, Y. Huang, and G. Tian, "A length ratio based neural network energy management strategy for online control of plug-in hybrid electric city bus," Applied Energy, vol. 177, pp. 71-80, 2016, doi: 10.1016/j.apenergy.2016.05.086.

[35] Y. Li, H. He, J. Peng, and H. Zhang, "Power Management for a Plug-in Hybrid Electric Vehicle Based on Reinforcement Learning with Continuous State and Action Spaces," Energy Procedia, vol. 142, pp. 2270-2275, 2017, doi: 10.1016/j.egypro.2017.12.629.

[36] Z. Chen, H. Hu, Y. Wu, R. Xiao, J. Shen, and Y. Liu, "Energy Management for a Power-Split Plug-In Hybrid Electric Vehicle Based on Reinforcement Learning," Applied Sciences, vol. 8, no. 12, 2018, doi: 10.3390/app8122494.

[37] L. Tribioli and S. Onori, "Analysis of energy management strategies in plug-in hybrid electric vehicles: Application to the GM Chevrolet Volt," in 2013 American Control Conference, 2013: IEEE, pp. 5966-5971.

[38] M. Morchid, "Parsimonious memory unit for recurrent neural networks with application to natural language processing," Neurocomputing, vol. 314, pp. 48-64, 2018/11/07/ 2018, doi: 10.1016/j.neucom.2018.05.081.

[39] S. Xie, H. He, and J. Peng, "An energy management strategy based on stochastic model predictive control for plug-in hybrid electric buses," Applied Energy, vol. 196, pp. 279-288, 2017, doi: 10.1016/j.apenergy.2016.12.112.

[40] X. Li, L. Han, H. Liu, W. Wang, and C. Xiang, "Real-time optimal energy management strategy for a dual-mode power-split hybrid electric vehicle based on an explicit model predictive control algorithm," Energy, vol. 172, pp. 1161-1178, 2019, doi: 10.1016/j.energy.2019.01.052. 
[41] Z. Chen, Y. Wu, N. Guo, J. Shen, and R. Xiao, "Rule-Based Online Energy Management Strategy for Power-Split Plug-In Hybrid Electric Vehicles," in 2018 IEEE Vehicle Power and Propulsion Conference (VPPC), 27-30 Aug. 2018 2018, pp. 1-6, doi: 10.1109/VPPC.2018.8605046.

[42] S. Xie, X. Hu, S. Qi, and K. Lang, "An artificial neural network-enhanced energy management strategy for plugin hybrid electric vehicles," Energy, vol. 163, pp. 837-848, 2018, doi: 10.1016/j.energy.2018.08.139.

[43] C. Yang, X. Jiao, L. Li, Y. Zhang, and Z. Chen, "A robust $\mathrm{H} \infty$ control-based hierarchical mode transition control system for plug-in hybrid electric vehicle," Mechanical Systems and Signal Processing, vol. 99, pp. 326-344, 2018, doi: 10.1016/j.ymssp.2017.06.023. 